\title{
ALGEBRAIC LOGIC, III. PREDICATES, TERMS, AND OPERATIONS IN POLYADIC ALGEBRAS
}

\author{
BY
}

\author{
PAUL R. HALMOS
}

Introduction. The theory of polyadic algebras is an algebraic photograph of the logical theory of first-order functional calculi. It is known, for instance, that the Gödel completeness theorem can be formulated in algebraic language as a representation theorem for a large class of simple polyadic algebras, together with the statement that every polyadic algebra is semisimple. The next desideratum is an algebraic study of the celebrated Gödel incompleteness theorem. Before that can be achieved, it is necessary to investigate the algebraic counterparts of some fundamental logical concepts (such as the ones mentioned in the title above). The purpose of this paper is to report the results of such an investigation( $\left.{ }^{1}\right)$.

Although this is the third of a sequence of papers on algebraic logic, its development does not lean very heavily on the first two papers. The main purpose of the first paper was to study the topological properties of quantification, via the Stone duality theory for Boolean algebras [Algebraic logic I, Compositio Math. vol. 12 (1955) pp. 217-249]. The purpose of the second paper was to study the algebraic properties of polyadic algebras, with main emphasis on their representation theory [Algebraic logic II, to appear in Fund. Math.] $\left.{ }^{2}\right)$. Since the methods and results of this paper might be described as combinatorial, neither the duality theory nor the representation theory plays any role; a sympathetic understanding of the basic definitions and of their elementary consequences is sufficient for present purposes. For the convenience of the reader, the basic definitions and theorems are summarized in $\$ 1$ below.

The most difficult concept introduced in this paper is the concept of a term ( $\$ 4)$, and the most difficult theorems are the ones that describe properties of terms ( $\$ 5-8)$ and the ones that give methods of constructing them $(\S \S 9,11$, and 13$)$. The climax is reached in $\S 9$; the existence theorem of that section (despite its somewhat complicated statement) turns out to be a most efficient tool for constructing terms satisfying various conditions. The remaining sections are devoted to auxiliary matters and to the more easily

Received by the editors January 5, 1956.

(1) The work on this paper was sponsored in part by the National Science Foundation, NSF grant 2266. Most of these results were announced in a note in the Proceedings of the National Academy of Sciences; cf. vol. 42 (1956) pp. 130-136.

(2) These papers will be referred to by the corresponding Roman numerals. For a brief summary of their contents, see Polyadic Boolean algebras, Proc. Nat. Acad. Sci. U.S.A. vol. 40 (1954) pp. 296-301. 
treated algebraic theory of predicates and operations. The applications (e.g., to the theory of equality in polyadic algebras and, eventually, to the algebraic version of the incompleteness theorem) will be published in subsequent papers of the sequence.

1. Polyadic algebras. The set-theoretic notation to be used (e.g., $u, n, c$, and $\epsilon$ ) is the standard one. The empty set is denoted by $\varnothing$, and nonbelonging by $\epsilon^{\prime}$, so that, for instance, $x \epsilon^{\prime} \varnothing$ for all $x$. A singleton (i.e., a set consisting of a single element, say $x$ ) is denoted by $\{x\}$; more generally, braces are employed, as always, to indicate the set of elements described within them. Sometimes, for the sake of notational simplicity, the braces are omitted from a symbol such as $\{x\}$. This is done mostly on the occasions when $\{x\}$ appears as an argument of a function; it is only rarely that the practice of writing $f(x)$ for $f(\{x\})$ can lead to confusion.

We shall be working with a fixed set $I$, and, unless there is explicit warning to the contrary, we shall assume that set means a subset of $I$ and transformation means a transformation (not necessarily one-to-one and not necessarily onto) from $I$ into itself. If a transformation does map $I$ onto itself in a oneto-one manner, we shall call it a permutation. Certain special transformations recur sufficiently often to deserve special symbols. Thus the identity transformation will be denoted by $\delta$, so that $\delta i=i$ for all $i$ in $I$. If $i$ and $j$ are in $I$, the transposition that interchanges them will be denoted by $(i, j)$, so that $(i, j)$ is the transformation such that $(i, j) i=j,(i, j) j=i$, and $(i, j) k=k$ whenever $k \epsilon^{\prime}\{i, j\}$. Clearly $\delta$ and $(i, j)$ are permutations.

If $i_{1}, \cdots, i_{n}$ are distinct elements of $I$, and if $j_{1}, \cdots, j_{n}$ are arbitrary (not necessarily distinct) elements of $I$, the symbol $\left(i_{1}, \cdots, i_{n} / j_{1}, \cdots, j_{n}\right)$ denotes the transformation that maps each $i$ onto the corresponding $j$ and everything else onto itself. Thus, in particular, if $i$ and $j$ are in $I$, then $(i / j)$ is the transformation such that $(i / j) i=j$ and $(i / j) k=k$ whenever $k \neq i$. This special case can be generalized in a slightly different direction: if $K \subset I$ and $j \in I$, we shall write $(K / j)$ for the transformation that maps every element of $K$ onto $j$ and every element of $I-K$ onto itself.

We shall say that a transformation $\tau$ lives on a set $J$ if $\tau J \subset J$ and if $\tau=\delta$ outside $J$. A transformation is finite if it lives on some finite set. Sometimes we shall consider a transformation, say $\tau^{-}$, on a subset $I^{-}$of $I$ (i.e., a transformation of $I^{-}$into itself); we shall then say that the canonical extension of $\tau^{-}$is the transformation $\tau$ on $I$ such that $\tau=\tau^{-}$on $I^{-}$and $\tau=\delta$ on $I-I^{-}$.

The following notation will be used for every Boolean algebra $A$ : the supremum of two elements $p$ and $q$ of $\boldsymbol{A}$ is $p \vee q$, the infimum of $p$ and $q$ is $p^{\wedge} q$, the complement of $p$ is $p^{\prime}$, the zero element of $\boldsymbol{A}$ is 0 , and the unit element of $\boldsymbol{A}$ is 1 . The natural order relation is denoted by $\leqq$, so that $p \leqq q$ means that $p \vee q=q$ (or, equivalently, that $p \wedge q=p$ ).

A quantifier (more precisely, an existential quantifier) on a Boolean algebra $\boldsymbol{A}$ is a mapping $\exists$ of $\boldsymbol{A}$ into itself such that 


$$
\begin{aligned}
\exists 0 & =0, \\
p & \leqq \exists p, \\
\exists(p \wedge \exists q) & =\exists p \wedge \exists q,
\end{aligned}
$$

for all $p$ and $q$ in $\boldsymbol{A}$. Suppose that $\boldsymbol{A}$ is a Boolean algebra, $I$ is a set, $\mathbf{S}$ is a mapping that associates a Boolean endomorphism $\mathbf{S}(\tau)$ of $A$ with every transformation $\tau$ on $I$, and $\exists$ is a mapping that associates a quantifier $\exists(J)$ on $A$ with every subset $J$ of $I$. The quadruple $(\boldsymbol{A}, I, \mathbf{S}, \exists)$ is a polyadic algebra if

(P1) $\exists(\varnothing)$ is the identity mapping on $A$,

(P2) $\exists(J \cup K)=\exists(J) \exists(K)$ for all $J$ and $K$,

(P3) $\mathbf{S}(\delta)$ is the identity mapping on $A$,

(P4) $\quad \mathbf{S}(\sigma \tau)=\mathbf{S}(\sigma) \mathbf{S}(\tau)$ for all $\sigma$ and $\tau$,

(P5) $\mathbf{S}(\sigma) \exists(J)=\mathbf{S}(\tau) \exists(J)$ whenever $\sigma=\tau$ outside $J$,

(P6) $\quad \exists(J) \mathbf{S}(\tau)=\mathbf{S}(\tau) \exists\left(\tau^{-1} J\right)$ whenever $\tau$ is such that it never maps two distinct elements of $I$ onto the same element of $J$.

It is often convenient to be slightly elliptical, and, instead of saying that $(\boldsymbol{A}, I, \mathbf{S}, \exists)$ is a polyadic algebra, to say that $\boldsymbol{A}$ is a polyadic algebra, or, alternatively, to say that $\boldsymbol{A}$ is an $I$-algebra. An element of $I$ is called a variable of the algebra $\boldsymbol{A}$. The degree of $\boldsymbol{A}$ is the cardinal number of the set of its variables.

An element $p$ of an $I$-algebra $A$ is independent of a subset $J$ of $I$ if $\exists(J) p$ $=p$; the set $J$ is a support of $p$ (or $J$ supports $p$ ) if $p$ is independent of $I-J$. The following facts about supports are easy to prove; we shall of ten use them without any explicit reference. (i) If $J$ supports $p$ and if $J \subset K$, then $K$ supports $p$. (ii) If $J$ supports $p$, then $J$ supports $p^{\prime}$; if $J$ supports $p$ and $q$, then $J$ supports $p \vee q$. (iii) If $J$ supports $p$, then $\exists(K) p=\exists(K \cap J) p$ for all $K$. (iv) If $J$ supports $p$, then $\tau J$ supports $\mathbf{S}(\tau) p$ for all $\tau$.

An $I$-algebra $\boldsymbol{A}$ is locally finite if each of its elements has a finite support. It is a fortunate circumstance that the logically most important polyadic algebras are the ones most amenable to algebraic treatment. These algebras are characterized by two conditions that pull against each other in a certain sense. The first condition is that their degree be infinite (i.e., that there be infinitely many variables); the second condition is that they be locally finite (i.e., that no single element depend on more than finitely many variables). We hereby establish a notation and an assumption that will be used throughout: we shall always assume that $(\boldsymbol{A}, I, \mathbf{S}, \exists)$ is a locally finite polyadic algebra of infinite degree.

Suppose that $c$ is a mapping that associates a Boolean endomorphism $c(J)$ of $\boldsymbol{A}$ with every subset $J$ of $I$. The mapping $c$ is a constant of the algebra $A$ if 


$$
\begin{aligned}
& c(\varnothing) \text { is the identity mapping on } A, \\
& c(J \cup K)=c(J) c(K), \\
& c(J) \exists(K)=\exists(K) c(J-K), \\
& \exists(J) c(K)=c(K) \exists(J-K), \\
& c(J) \mathbf{S}(\tau)=\mathbf{S}(\tau) c\left(\tau^{-1} J\right),
\end{aligned}
$$

whenever $J$ and $K$ are subsets of $I$ and $\tau$ is a transformation on $I$. We shall need to make use of the following three facts about constants.

(1.1) THEOREM. [II, (12.3)]. If $c$ is a mapping from all finite subsets of I into Boolean endomorphisms of $A$ satisfying (C1)-(C5) whenever $J$ and $K$ are finite subsets of $I$ and $\tau$ is a finite transformation on $I$, then there exists a unique constant $\tilde{c}$ of $\boldsymbol{A}$ such that $\tilde{c}(J)=c(J)$ for all finite subsets $J$ of $I$.

(1.2) Theorem. [II, (14.1)]. If $f$ is a Boolean endomorphism of $\boldsymbol{A}$ and if $\boldsymbol{i}$ is an element of $I$ such that

$$
\begin{aligned}
& \exists(i) f=f, \\
& f \exists(i)=\exists(i), \\
& f \exists(j)=\exists(j) f
\end{aligned}
$$

whenever $j \neq i$,

then there exists a unique constant $c$ of $\boldsymbol{A}$ such that $c(i)=f$.

(1.3) Theorem. [II, (16.2)]. If $b$ and $c$ are (not necessarily distinct) constants and if $J$ and $K$ are disjoint subsets of $I$, then the Boolean endomorphisms $b(J)$ and $c(K)$ commute with each other.

Associated with every subset $I^{-}$of $I$ there is a polyadic algebra $\left(\mathrm{A}^{-}, \mathrm{I}^{-}\right.$, $\left.\mathbf{S}^{-}, \Xi^{-}\right)$that is said to be obtained from $A$ by fixing the variables of $I-I^{-}$. The Boolean algebra $\boldsymbol{A}^{-}$is (by definition) the same as the Boolean algebra $\boldsymbol{A}$. To define $\mathbf{S}^{-}$, suppose that $\tau^{-}$is a transformation on $I^{-}$, let $\tau$ be its canonical extension to $I$, and write $\mathbf{S}^{-}\left(\tau^{-}\right)=\mathbf{S}(\tau)$; to define $\Xi^{-}$, suppose that $J$ is a subset of $I^{-}$, and write $\exists^{-}(J)=\exists(J)$. The following result establishes a connection between this concept and the preceding one; it says, in effect, that a fixed variable is a constant.

(1.4) Theorem. [II, §15]. Suppose that $I^{-} \subset I$ and that $\left(\mathrm{A}^{-}, \mathrm{I}^{-}, \mathrm{S}^{-}, \mathrm{\Xi}^{-}\right)$ is the algebra obtained from $A$ by fixing the variables of $I-I^{-}$. If $j \in I-I^{-}$and if $c^{-}(J)=\mathbf{S}(J / j)$ for every subset $J$ of $I^{-}$, then $c^{-}$is a constant of $\mathbf{A}^{-}$.

2. Predicates. The elements of a polyadic algebra are the algebraic counterparts of propositional functions. In a suggestive and reasonably flexible terminology, an element with support $J$ will be called a $J$-proposition. If $J$ is empty, a $J$-proposition (i.e., a $\varnothing$-proposition) will be called a closed proposition, or, simply a proposition. 
There is a distinction, in ordinary logical parlance, between a "propositional function" and a "predicate" ; that distinction can be profitably imitated in the theory of polyadic algebras. In the usual treatment of first-order functional calculi a predicate symbol all by itself is not a well-formed formula; it becomes one, however, when it is followed by the appropriate number of individual variables. This suggests that in the algebraic theory a predicate should be a function whose arguments are variables and whose values are elements of a polyadic algebra. Not every such function deserves to be called a predicate; the general definition must somehow take account of special facts such as that if in $x=y$ the variables $x$ and $y$ are replaced by $u$ and $v$ respectively, then the result is $u=v$. These considerations motivate the following definition.

An $n$-place (or $n$-ary) predicate $(n=1,2,3, \cdots)$ of an $I$-algebra $A$ is a function $P$ from $I^{n}$ into $A$ such that if $\left(i_{1}, \cdots, i_{n}\right) \in I^{n}$ and if $\tau$ is a transformation on $I$, then

$$
\mathbf{S}(\tau) P\left(i_{1}, \cdots, i_{n}\right)=P\left(\tau i_{1}, \cdots, \tau i_{n}\right) .
$$

We shall sometimes say that an $n$-place predicate is a predicate of degree $n$. The basic theory of predicates is quite easy; there are only two main facts that it is useful to know. Theorem (2.2) asserts that the variables that a particular value of a predicate depends on are apparent at a glance. An $n$-place predicate and an element of $I^{n}$ together define an element of $A$; Lemma (2.3) gives a sort of converse for this assertion.

(2.2) THEOREM. If $P$ is an $n$-place predicate and if $\left(i_{1}, \cdots, i_{n}\right) \in I^{n}$, then $\left\{i_{1}, \cdots, i_{n}\right\}$ supports $P\left(i_{1}, \cdots, i_{n}\right)$.

Proof. In view of the local finiteness of $A$, it is sufficient to show that $\exists(j) P\left(i_{1}, \cdots, i_{n}\right)=P\left(i_{1}, \cdots, i_{n}\right)$ whenever $j \epsilon^{\prime}\left\{i_{1}, \cdots, i_{n}\right\}$. If $k$ is any variable distinct from $j$, then

$$
\begin{aligned}
\exists(j) P\left(i_{1}, \cdots, i_{n}\right)=\exists(j) \mathbf{S}(j / k) P\left(i_{1}, \cdots, i_{n}\right)[\text { by }(2.1)] \\
=\mathbf{S}(j / k) P\left(i_{1}, \cdots, i_{n}\right)[\text { since } j \neq k]=P\left(i_{1}, \cdots, i_{n}\right)[\text { by }(2.1)] .
\end{aligned}
$$

(2.3) Lemma. If $p \in A$ and if $j_{1}, \cdots, j_{n}$ are distinct elements of $I$ such that $\left\{j_{1}, \cdots, j_{n}\right\}$ supports $p$, then there exists a unique $n$-place predicate $P$ of $\boldsymbol{A}$ such that $P\left(j_{1}, \cdots, j_{n}\right)=p$.

Proof. The predicate $P$ is defined by

$$
P\left(i_{1}, \cdots, i_{n}\right)=\mathbf{S}\left(j_{1}, \cdots, j_{n} / i_{1}, \cdots, i_{n}\right) p .
$$

To prove that the function $P$ is indeed a predicate, let $\tau$ be an arbitrary transformation on $I$ and note that if $j \in\left\{j_{1}, \cdots, j_{n}\right\}$, then

$$
\tau \cdot\left(j_{1}, \cdots, j_{n} / i_{1}, \cdots, i_{n}\right) j=\left(j_{1}, \cdots, j_{n} / \tau i_{1}, \cdots, \tau i_{n}\right) j .
$$

Since, by assumption, $\left\{j_{1}, \cdots, j_{n}\right\}$ supports $p$, it follows that 


$$
\begin{aligned}
\mathbf{S}(\tau) P\left(i_{1}, \cdots, i_{n}\right) & =\mathbf{S}(\tau) \mathbf{S}\left(j_{1}, \cdots, j_{n} / i_{1}, \cdots, i_{n}\right) p \\
& =\mathbf{S}\left(j_{1}, \cdots, j_{n} / \tau i_{1}, \cdots, \tau i_{n}\right) p=P\left(\tau i_{1}, \cdots, \tau i_{n}\right) .
\end{aligned}
$$

The uniqueness of $P$ is an immediate consequence of (2.1), with

$$
\left(j_{1}, \cdots, j_{n} / i_{1}, \cdots, i_{n}\right)
$$

in the role of $\tau$.

(2.4) CoRollary. Every element of $A$ is in the range of some predicate of $A$.

Whereas (2.2) guarantees that a value of a predicate depends on no more variables than the visible ones, it may well happen that it depends on fewer. Suppose, for example, that $Q$ is a 1-place (unary) predicate and write

$$
P(i, j)=Q(i)
$$

whenever $i$ and $j$ are variables. If $\tau$ is an arbitrary transformation on $I$, then

$$
\mathbf{S}(\tau) P(i, j)=\mathbf{S}(\tau) Q(i)=Q(\tau i)=P(\tau i, \tau j),
$$

so that $P$ is a 2 -place (binary) predicate. By (2.2), $\{i, j\}$ supports $P(i, j)$ for each $i$ and $j$; since, however, $P(i, j)=Q(i)$, an application to (2.2) to $Q$ shows that in fact $\{i\}$ also supports $P(i, j)$.

The preceding paragraph suggests a possible generalization of the concept of predicate: we may ask what happens if the roles of $P$ and $Q$ are interchanged. If, in other words, $Q$ is a binary predicate, and if $j$ is some particular variable, we may write

$$
P(i)=Q(i, j)
$$

for every variable $i$. If $\tau$ is a transformation on $I$ such that $\tau j=j$, then

$$
\mathbf{S}(\tau) P(i)=\mathbf{S}(\tau) Q(i, j)=Q(\tau i, j)=P(\tau i) .
$$

If, however, $\tau j \neq j$, then $\mathbf{S}(\tau) P(i)$ is not necessarily the same as $P(\tau i)$. If, in particular, $\tau=(i, j)$, then $\mathbf{S}(\tau) P(i)=Q(j, i)$, whereas $P(\tau i)=Q(j, j)$, and, in general, $Q(j, i) \neq Q(j, j)$. If, similarly, $\tau=(j / i)$, then $\mathbf{S}(\tau) P(j)=Q(i, i)$, whereas $P(\tau j)=Q(i, j)$, and, in general, $Q(i, i) \neq Q(i, j)$.

A study of this phenomenon shows that the appropriate general concept is defined as follows. Let $J$ be an arbitrary finite subset of $I$, write $I^{-}=I-J$, and let $\left(A^{-}, I^{-}, \mathbf{S}^{-}, \exists^{-}\right)$be the polyadic algebra obtained from $A$ by fixing the variables of $J$; define an $n$-place $J$-predicate of $A$ as an $n$-place predicate of $A^{-}$. Equivalently, an $n$-place $J$-predicate of $A$ is a function $P$ from $(I-J)^{n}$ into $A$ with the following property: if $\tau^{-}$is a transformation on $I-J$ and if $\tau$ is its canonical extension to $I$, then

$$
\mathbf{S}(\tau) P\left(i_{1}, \cdots, i_{n}\right)=P\left(\tau i_{1}, \cdots, \tau i_{n}\right)
$$

for every element $\left(i_{1}, \cdots, i_{n}\right)$ of $(I-J)^{n}$. The extent to which $J$-predicates generalize predicates is clear: the special concept is obtained from the general 
one by setting $J$ equal to $\varnothing$. In other words, what was called a predicate before, is a $\varnothing$-predicate in the present terminology. Note also that the relation of $J$-predicates to predicates is the same as the relation of $J$-propositions to propositions.

The following result has the effect of reducing the theory of $J$-predicates to the theory of ordinary predicates.

(2.5) Theorem. Suppose that $J$ is a finite subset of $I$; let $j_{1}, \cdots, j_{m}$ be the distinct elements of $J$. If $Q$ is an $(n+m)$-place predicate, and if

$$
P\left(i_{1}, \cdots, i_{n}\right)=Q\left(i_{1}, \cdots, i_{n}, j_{1}, \cdots, j_{m}\right)
$$

whenever $\left(i_{1}, \cdots, i_{n}\right) \in(I-j)^{n}$, then $P$ is an n-place $J$-predicate. If, conversely, $P$ is an $n$-place $J$-predicate, then there exists a unique $(n+m)$-place predicate $Q$ such that (2.6) holds for all $\left(i_{1}, \cdots, i_{n}\right)$ in $(I-J)^{n}$.

Proof. The first conclusion is an immediate consequence of the definition of a $J$-predicate; it is necessary to prove the converse only. For this purpose, write $I^{-}=I-J$ and let $\left(\boldsymbol{A}^{-}, I^{-}, \mathbf{S}^{-}, \boldsymbol{\Xi}^{-}\right)$be the algebra obtained from $\boldsymbol{A}$ by fixing the variables of $J$. If $\left(i_{1}, \cdots, i_{n}\right) \in(I-J)^{n}$, then it follows from (2.2) that $\left\{i_{1}, \cdots, i_{n}\right\}$ supports $P\left(i_{1}, \cdots, i_{n}\right)$ in $A^{-}$. This means that if $i \epsilon I-J$ and $i \epsilon^{\prime}\left\{i_{1}, \cdots, i_{n}\right\}$, then $\exists(i) P\left(i_{1}, \cdots, i_{n}\right)=P\left(i_{1}, \cdots, i_{n}\right)$, and this, in turn, implies that $\left\{i_{1}, \cdots, i_{n}, j_{1}, \cdots, j_{m}\right\}$ supports $P\left(i_{1}, \cdots, i_{n}\right)$ in $A$. If, in particular, $k_{1}, \cdots, k_{n}$ are distinct elements of $I-J$, then it follows from (2.3) that there exists a unique $(n+m)$-place predicate $Q$ such that

$$
P\left(k_{1}, \cdots, k_{n}\right)=Q\left(k_{1}, \cdots, k_{n}, j_{1}, \cdots, j_{m}\right) .
$$

Suppose now that $\left(i_{1}, \cdots, i_{n}\right) \epsilon(I-J)^{n}$ and let $\tau^{-}$be the transformation $\left(k_{1}, \cdots, k_{n} / i_{1}, \cdots, i_{n}\right)$ on $I-J$. If $\tau$ is the canonical extension of $\tau^{-}$to $I$, then

$$
\begin{aligned}
Q\left(i_{1}, \cdots, i_{n}, j_{1}, \cdots, j_{m}\right)=Q\left(\tau k_{1}, \cdots, \tau k_{n}, \tau j_{1}, \cdots, \tau j_{m}\right) \\
=\mathbf{S}(\tau) Q\left(k_{1}, \cdots, k_{n}, j_{1}, \cdots, j_{m}\right)=\mathbf{S}(\tau) P\left(k_{1}, \cdots, k_{n}\right) \\
=\mathbf{S}^{-}\left(\tau^{-}\right) P\left(k_{1}, \cdots, k_{n}\right)=P\left(\tau^{-} k_{1}, \cdots, \tau^{-} k_{n}\right)=P\left(i_{1}, \cdots i_{n}\right) .
\end{aligned}
$$

This completes the proof of the theorem.

3. Transformations of type $(M, N)$. The reason for restricting the discussion of polyadic algebras to algebras of infinite degree is that the availability of a large supply of variables of ten makes it possible to avoid unpleasant notational collisions. In this section we introduce a special class of transformations designed to exploit the availability of infinitely many variables; using these transformations we shall be able to avoid most collisions in an almost automatic manner.

Suppose that $M$ and $N$ are finite subsets of $I$. A transformation $\sigma$ on $I$ will be said to be of type $(M, N)$ if 


$$
\left\{\begin{array}{l}
\sigma \text { is one-to-one on } M, \\
\sigma M \subset I-(M \cup N), \\
\sigma=\delta \text { outside } M .
\end{array}\right.
$$

If $\sigma$ is of type $(M, N)$, we shall denote by $\tilde{\sigma}$ the transformation that acts as the inverse of $\sigma$ in $\sigma M$ and is equal to $\delta$ outside $\sigma M$; in other words,

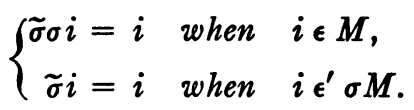

A straightforward application of the definitions (3.1) and (3.2) shows that

$$
\tilde{\sigma} \sigma=\tilde{\sigma} \text {. }
$$

When a transformation is used to avoid variable collisions, it is often necessary to prove that the result is independent of the particular choice of that transformation. The following lemma is the most useful tool in such unambiguity proofs.

(3.4) LeMmA. If both $\sigma$ and $\tau$ are transformations of type $(M, N)$, then there exists a permutation $\pi$ on $I$ such that

$$
\begin{aligned}
\pi \tau & =\sigma \pi, \\
\pi & =\delta \quad \text { outside } \quad \tau M \cup \sigma M .
\end{aligned}
$$

If $\pi$ is a permutation satisfying (3.5) and (3.6), then

$$
\pi \tilde{\tau}=\tilde{\sigma} \pi \text {. }
$$

Proof. Since $\sigma M-\tau M$ has the same (finite) number of elements as $\tau M-\sigma M$, there exists a one-to-one mapping $\pi$ from $\sigma M-\tau M$ onto $\tau M-\sigma M$; extend $\pi$ to $I$ by writing

$$
\pi=\sigma \tilde{\tau} \text { on } \tau M \text { and } \pi=\delta \text { outside } \sigma M \cup \tau M \text {. }
$$

(i) If $j \in M$, then $\pi \tau j=\sigma \tilde{\tau} \tau j=\sigma j$ and $\sigma \pi j=\sigma j$. (ii) If $j \in \tau M$, then $\pi \tau j=\pi j=\sigma \tilde{\tau} j$ and $\sigma \pi j=\sigma \sigma \tilde{\tau} j=\sigma \tilde{\tau} j$. (iii) If $j \epsilon \sigma M-\tau M$, then $\pi \tau j=\pi j$ and $\sigma \pi j=\pi j$. (iv) If, finally, $j \epsilon^{\prime} M \cup \sigma M \cup \tau M$, then $\pi \tau j=\pi j=j$ and $\sigma \pi j=\sigma j=j$. This proves the first assertion of the lemma.

To prove the second assertion, suppose first that $j \in \tau M$, so that $j=\tau i$ with $i$ in $M$. It follows that $\pi \tilde{\tau} j=\pi \tilde{\tau} \tau i=\pi i=i$ and $\tilde{\sigma} \pi j=\tilde{\sigma} \pi \tau i=\tilde{\sigma} \sigma \pi i=\tilde{\sigma} \sigma i=i$. If, on the other hand, $j \epsilon^{\prime} \tau M$, then $\pi j \epsilon^{\prime} \pi \tau M=\sigma \pi M=\sigma M$. Since $j \epsilon^{\prime} \tau M$, it follows that $\pi \tilde{\tau} j=\pi j$; since $\pi j \epsilon^{\prime} \sigma M$, it follows that $\tilde{\sigma} \pi j=\pi j$. This completes the proof of the lemma.

4. Terms. Propositions, $J$-propositions, predicates, and $J$-predicates all have to do with the elements of a polyadic algebra. In many treatments they are even denoted by similar symbols, namely by functional symbols that must be followed by a certain number of arguments before they take on a specific 
meaning. They are thus, roughly speaking, the concepts whose symbols stand outside the parentheses in the functional notation. What are the things that stand inside those parentheses? By what, in other words, may we replace the variables that a $J$-proposition or a predicate depends on? One answer is: by constants; another answer is: by variables. There is still another answer. There is a concept that includes both constants and variables, a general concept that stands in the same relation to constants as $J$-propositions stand to propositions. The new concept is that of a term, or, more explicitly, a $J$-term.

To understand the definition of a term, it is advisable first to introduce the auxiliary concept of a $J$-constant, or, roughly speaking, a "variable constant." Suppose that $J$ is a finite subset of $I$. If $I^{-}=I-J$, and if $A^{-}$is the $I^{-}$-algebra obtained from $A$ by fixing the variables of $J$, then a $J$-constant of $\boldsymbol{A}$ is defined to be a constant of the algebra $\boldsymbol{A}^{-}$. Observe that a $\varnothing$-constant is simply a constant.

If $c$ is a $J$-constant, we shall denote the value of $c$ at a subset $K$ of $I-J$ by $\mathbf{S}(K / c)$. (This notation is different from the one used before for constants; the innovation has a very beneficial unifying and simplifying effect.) The symbol $\mathbf{S}(K / c)$ denotes thus a Boolean endomorphism of $\boldsymbol{A}$. Using this notation, and recalling the definitions of a constant and of the process of fixing variables, we may express the definition of a $J$-constant as follows. A $J$-constant is a mapping $c$ that associates with every subset $K$ of $I-J$ a Boolean endomorphism of $\boldsymbol{A}$, to be denoted by $\mathbf{S}(K / c)$, so that

$$
\mathbf{S}(\varnothing / c) \text { is the identity mapping on } A \text {, }
$$

$$
\begin{aligned}
\mathbf{S}(H \cup K / c) & =\mathbf{S}(H / c) \mathbf{S}(K / c), \\
\mathbf{S}(H / c) \exists(K) & =\exists(K) \mathbf{S}(H-K / c), \\
\exists(H) \mathbf{S}(K / c) & =\mathbf{S}(K / c) \exists(H-K), \\
\mathbf{S}(K / c) \mathbf{S}(\tau) & =\mathbf{S}(\tau) \mathbf{S}\left(\tau^{-1} K / c\right),
\end{aligned}
$$

whenever $H$ and $K$ are subsets of $I-J$ and $\tau$ is a transformation that lives on $I-J$.

We have already observed that every constant is a $\varnothing$-constant. Constants can also be used to obtain examples of $J$-constants; in fact it might be said that a constant is automatically a $J$-constant (for every finite set $J$ ). This is not quite accurate; what should be said is that the restriction of a constant to the subsets of $I-J$ is a $J$-constant. These examples are rather trivial. A nontrivial example is obtained from variables. If $j$ is an element of $I$ and if $c_{j}$ denotes the mapping (from subsets of $I-\{j\}$ to Boolean endomorphisms of $\boldsymbol{A})$ that assigns to a subset $K$ of $I-\{j\}$ the value $\mathbf{S}(K / j)$, then $c_{j}$ is a $j$ constant (i.e., a $\{j\}$-constant); cf. (1.4)). The distinction between $j$ and $c_{j}$ is not always easy to maintain; we shall sometimes speak as if $j$ itself were a $j$-constant. 
If $c$ is a $J$-constant, then $\mathbf{S}(K / c)$ is not defined for every subset $K$ of $I$; this is the main point in which the concept of a $J$-term differs from that of a $J$-constant. A $J$-term (where $J$ is a finite subset of $I$ ) is a mapping $t$ that associates with every subset $K$ of $I$ a Boolean endomorphism $\mathbf{S}(K / t)$ of $A$, so that the restriction of $t$ to the subsets of $I-J$ is a $J$-constant of $A$, and so that

$$
\mathbf{S}(K / t) p=\mathbf{S}(\sigma K / t) \mathbf{S}(\sigma) p
$$

whenever $p$ is an element of $\boldsymbol{A}$ with finite support $L, K$ is a finite subset of $I$, and $\sigma$ is a transformation of type $(K, L \cup J)$. The reason for the restriction (4.1) can be illustrated by borrowing the notation from the subsequent theory of "terms in predicates." If $P$ is a binary predicate and if $L=\{i, j\}$, then $L$ supports $p=P(i, j)$. If $K=\{j, k\}$ (with $i \epsilon^{\prime} K$ ), and if $\sigma$ is a transformation of type $(K, L \cup J)$, then $\sigma i=i$. Since both $\mathbf{S}(K / t) P(i, j)$ and $\mathbf{S}(\sigma K / t) \mathbf{S}(\sigma) P(i, j)$ are equal to $P(i, t)$, the assertion of equation (4.1) agrees with our intuitive expectations. The idea is simply this: to replace the variables of $K$ by $t$ in $p$, first transform them into some neutral variables far from the scene of action (and, in particular, not in $J$ ), and then replace those new variables by the $J$ constant that $t$ reduces to out there.

It is sometimes useful to know that if $J_{1}$ and $J_{2}$ are finite subsets of $I$ such that $J_{1} \subset J_{2}$, then every $J_{1}$-term is a $J_{2}$-term. Suppose, indeed, that $t$ is a $J_{1}$-term. Since $I-J_{2} \subset I-J_{1}$, it follows that the restriction of $t$ to the subsets of $I-J_{2}$ is a $J_{2}$-constant. If, moreover, $K$ and $L$ are finite subsets of $I$, then every transformation of type $\left(K, L \cup J_{2}\right)$ is automatically a transformation of type $\left(K, L \cup J_{1}\right)$; the assertion that $t$ is a $J_{2}$-term now follows immediately from the definition.

The condition (4.1) in the definition of a $J$-term is very strong; $a b$ initio it is not even clear that any nontrivial $J$-terms exist. Trivial examples can be obtained from constants. A constant $c$ is a $J$-term (for every finite set $J$ ), but even this takes some proving. The proof of (4.1) runs as follows. If $p$ has finite support $L$, if $K$ is a finite subset of $I$, and if $\sigma$ is a transformation of type $(K, L \cup J)$, then

$$
\begin{aligned}
\mathbf{S}(\sigma & K / c) \mathbf{S}(\sigma) p=\mathbf{S}(\sigma) \mathbf{S}\left(\sigma^{-1} \sigma K / c\right) p[\text { by (C5)] } \\
& =\mathbf{S}(\sigma) \mathbf{S}(K \cup \sigma K / c) p[\text { by (3.1)] }=\mathbf{S}(\sigma) \mathbf{S}(K / c) \mathbf{S}(\sigma K / c) p[\text { by (C2)] } \\
& =\mathbf{S}(\sigma) \mathbf{S}(K / c) p[\text { by (3.1), (C3), and (C1)] } \\
& =\mathbf{S}(K / c) p[\text { by (3.1) and (C4)]. }
\end{aligned}
$$

The strength of the definition will be useful to us; once we know that something is a $J$-term, we shall be able to make powerful use of (4.1). We shall presently prove the existence of many $J$-terms; we shall show, in fact, that every $J$-constant has a unique extension to a $J$-term. For this purpose we turn now to a systematic study of some of the elementary properties of $J$. constants. 


\section{5. $J$-constants.}

(5.1) Lemma. If $c$ is a J-constant, if $L$ supports $p$, and if $K \subset I-J$, then $J \mathbf{u}(L-K)$ supports $\mathbf{S}(K / c) p$.

Proof. If $i \epsilon^{\prime} J \cup(L-K)$, then either $i \epsilon^{\prime} J \cup K \cup L$ or $i \epsilon K$. If $i \epsilon^{\prime} J \cup K$ u $L$, then

$$
\exists(i) \mathbf{S}(K / c) p=\mathbf{S}(K / c) \exists(i) p[\text { by }(\mathrm{T} 4)]=\mathbf{S}(K / c) p ;
$$

if $i \in K$, then

$$
\exists(i) \mathbf{S}(K / c) p=\mathbf{S}(K / c) p[\text { by (T4)]. }
$$

(5.2) Lemma. If $c$ is a $J$-constant, if $L$ supports $p$, and if $K \subset I-J$, then

$$
\mathbf{S}(K / c) p=\mathbf{S}(K \cap L / c) p .
$$

Proof. Since $p$ is independent of $K-L$, it follows that

$$
\mathbf{S}(K / c) p=\mathbf{S}(K / c) \exists(K-L) p,
$$

and hence, by (T3), that

$$
\mathbf{S}(K / c) p=\exists(K-L) \mathbf{S}(K \cap L / c) p .
$$

By (5.1), $J \cup(L-K)$ supports $\mathbf{S}(K \cap L / c) p$; since this set is disjoint from $K-L$, it follows that $\mathbf{S}(K \cap L / c) p$ is independent of $K-L$, and hence that

$$
\mathbf{S}(K / c) p=\mathbf{S}(K \cap L / c) p .
$$

(5.3) Lemma. If $c$ is a $J$-constant, if $L$ is a finite support of $p$, if $K$ is a finite subset of $I$, and if $\sigma$ is a transformation of type $(K, L \cup J)$, then $J \cup(L-K)$ supports $\mathbf{S}(\sigma K / c) \mathbf{S}(\sigma) p$.

Proof. Since $L$ supports $p$, it follows that $\sigma L$ supports $\mathbf{S}(\sigma) p$, and therefore, by (5.1), that $J \cup(\sigma L-\sigma K)$ supports $\mathbf{S}(\sigma K / c) \mathbf{S}(\sigma) p$. Since

$$
\sigma L=\sigma(L \cap K) \cup \sigma(L-K) \subset \sigma K \cup(L-K),
$$

it follows that

$$
\sigma L-\sigma K \subset L-K .
$$

(5.4) Lemma. If $c$ is a $J$-constant, if $L$ is a finite support of $p$, if $K$ is a finite subset of $I$, and if both $\sigma$ and $\tau$ are transformations of type $(K, L \cup J)$, then

$$
\mathbf{S}(\sigma K / c) \mathbf{S}(\sigma) p=\mathbf{S}(\tau K / c) \mathbf{S}(\tau) p .
$$

Proof. Apply (3.4) to find a permutation $\pi$ with the properties there described, and replace $\mathbf{S}(\tau)$ in $\mathbf{S}(\tau K / c) \mathbf{S}(\tau) p$ by $\mathbf{S}\left(\pi^{-1}\right) \mathbf{S}(\sigma) \mathbf{S}(\pi)$. Since $\pi=\delta$ in $L$ and since $L$ supports $p$, the factor $\mathbf{S}(\pi)$ may be erased from the result. Since $\pi^{-1}$ lives on $I-J$ (in fact on $I-(L \cup J)$ ), (T5) is applicable; it yields 


$$
\mathbf{S}(\tau K / c) \mathbf{S}\left(\pi^{-1}\right)=\mathbf{S}\left(\pi^{-1}\right) \mathbf{S}(\boldsymbol{\pi} \tau K / c) .
$$

Since $\pi \tau K=\sigma \pi K=\sigma K$, it follows that

$$
\mathbf{S}(\tau K / c) \mathbf{S}(\tau) p=\mathbf{S}\left(\pi^{-1}\right) \mathbf{S}(\sigma K / c) \mathbf{S}(\sigma) p .
$$

Since, by (5.3), $J \cup L$ supports $\mathbf{S}(\sigma K / c) \mathbf{S}(\sigma) p$, and since $\pi^{-1}=\delta$ on $J \cup L$, the proof is complete.

(5.5) Lemma. If $c$ is a J-constant, if $L_{1}$ and $L_{2}$ are finite supports of $p$, if $K$ is a finite subset of $I$, and if $\sigma_{1}$ and $\sigma_{2}$ are transformations of types $\left(K, L_{1} \cup J\right)$ and $\left(K, L_{2} \cup J\right)$ respectively, then

$$
\mathbf{S}\left(\sigma_{1} K / c\right) \mathbf{S}\left(\sigma_{1}\right) p=\mathbf{S}\left(\sigma_{2} K / c\right) \mathbf{S}\left(\sigma_{2}\right) p .
$$

Proof. If $L=L_{1} \cup L_{2}$, then $L$ is a finite support of $p$; let $\tau$ be an arbitrary transformation of type $(K, L \cup J)$. (The proof that such a $\tau$ exists uses the assumption that $I$ is infinite.) It follows that $\tau$ is a transformation of type $\left(K, L_{1} \cup J\right)$ and also a transformation of type $\left(K, L_{2} \cup J\right)$; the desired result is now an immediate consequence of (5.4).

6. The extension of $J$-constants. Since a constant is determined by its values on finite sets, the same is true of a $J$-constant, and, therefore, of a $J$ term. For convenience of reference, we restate this informal comment as a lemma; the proof of the lemma is an easy application of the corresponding result (1.1) for constants.

(6.1) LemMA. If $J$ is a finite subset of $I$ and if $t$ is a mapping that associates with every finite subset $K$ of $I$ a Boolean endomorphism $\mathbf{S}(K / t)$ of $\boldsymbol{A}$ so that (T1)-(T5) are satisfied for finite subsets $H$ and $K$ of $I-J$ and for finite transformations $\tau$ that live on $I-J$ and so that $\mathbf{S}(K / t)=\mathbf{S}(\sigma K / t) \mathbf{S}(\sigma) p$ whenever $p$ is an element of $\boldsymbol{A}$ with finite support $L, K$ is a finite subset of $I$, and $\sigma$ is a transformation of type $(K, L \cup J)$, then there exists a unique $J$-term $\tilde{i}$ such that $\mathbf{S}(K / \tilde{t})=\mathbf{S}(K / t)$ for all finite subsets $K$ of $I$.

Once this result is available, there is not much point in maintaining the distinction between $t$ and $\tilde{t}$; in the sequel we shall use the same symbol (namely $t$ ) for both, and, correspondingly, we shall say that $t$ is a $J$-term (instead of saying that $t$ can be extended to infinite sets so as to become one).

Suppose now that $c$ is a $J$-constant; it is natural to try to extend $c$ to a $J$-term as follows. Given any element $p$ of $\boldsymbol{A}$ and any finite subset $K$ of $I$, let $L$ be a finite support of $p$, let $\sigma$ be a transformation of type $(K, L \cup J)$, and write

$$
\mathbf{S}(K / t) p=\mathbf{S}(\sigma K / c) \mathbf{S}(\sigma) p .
$$

It follows from (5.5) that this definition is unambiguous, i.e., that it does not depend on the particular choice of $L$ and of $\sigma$. We indicate the dependence of $t$ on $c$ by writing 


$$
t=\bar{c}
$$

we proceed to prove that $t$ has the properties it should have.

(6.4) Lemma. If $c$ is a $J$-constant, if $t=\bar{c}$, and if $K$ is a finite subset of $I$, then $\mathbf{S}(K / t)$ is a Boolean endomorphism of $\boldsymbol{A}$.

Proof. If $p \in A$, and if $L$ is a finite support of $p$, then $L$ supports $p^{\prime}$ also; it follows that if $\sigma$ is a transformation of type $(K, L \cup J)$, then

$$
\mathbf{S}(K / t) p^{\prime}=\mathbf{S}(\sigma K / c) \mathbf{S}(\sigma) p^{\prime}=(\mathbf{S}(\sigma K / c) \mathbf{S}(\sigma) p)^{\prime}=(\mathbf{S}(K / t) p)^{\prime} .
$$

If $p$ and $q$ are in $A$, let $L$ be a finite set that supports both of them, and let $\sigma$ be a transformation of type $(K, L \cup J)$. Since $L$ supports $p \vee q$, it follows that

$$
\begin{aligned}
\mathbf{S}(K / t)(p \vee q) & =\mathbf{S}(\sigma K / c) \mathbf{S}(\sigma)(p \vee q) \\
& =\mathbf{S}(\sigma K / c) \mathbf{S}(\sigma) p \vee \mathbf{S}(\sigma K / c) \mathbf{S}(\sigma) q=\mathbf{S}(K / t) p \vee \mathbf{S}(K / t) q .
\end{aligned}
$$

(6.5) Lemma. If $c$ is a $J$-constant, if $t=\bar{c}$, and if $K$ is a finite subset of $I-J$, then $\mathbf{S}(K / t)=\mathbf{S}(K / c)$.

Proof. This is similar to the proof that a constant is a $J$-term. If $p \in A$, if $L$ is a finite support of $p$, and if $\sigma$ is a transformation of type $(K, L \cup J)$, then

$$
\begin{aligned}
\mathbf{S}(K / t) p & =\mathbf{S}(\sigma K / c) \mathbf{S}(\sigma) p[\text { by }(6.2)]=\mathbf{S}(\sigma) \mathbf{S}\left(\sigma^{-1} \sigma K / c\right) p[\text { by }(\mathrm{T} 5)] \\
& =\mathbf{S}(\sigma) \mathbf{S}(K \cup \sigma K / c) p[\text { by }(3.1)]=\mathbf{S}(\sigma) \mathbf{S}(K / c) \mathbf{S}(\sigma K / c) p[\text { by (T2)] } \\
& =\mathbf{S}(\sigma) \mathbf{S}(K / c) p[\text { by (3.1), (T3), and (T1)] }=\mathbf{S}(K / c) p
\end{aligned}
$$

[by (3.1) and (T4)].

(6.6) Lemma. If $c$ is a $J$-constant, if $t=\bar{c}$, if $p$ is an element of $\boldsymbol{A}$ with $f$ nite support $L$, if $K$ is a finite subset of $I$, and if $\sigma$ is a transformation of type $(K, L \cup J)$, then

$$
\mathbf{S}(K / t) p=\mathbf{S}(\sigma K / t) \mathbf{S}(\sigma) p .
$$

Proof. By definition, $\mathbf{S}(K / t) p=\mathbf{S}(\sigma K / c) \mathbf{S}(\sigma) p$; the desired result follows from (6.5) (with $\sigma K$ in the role of $K$ ).

(6.7) THEOREM. If $c$ is a J-constant, then there exists a unique J-term $t$ (namely, $t=\bar{c}$ ) such that

$$
\mathbf{S}(K / t)=\mathbf{S}(K / c)
$$

whenever $K \subset I-J$.

Proof. The existence of $t$ is contained in the preceding four lemmas. Uniqueness follows from the fact that a term is uniquely determined by its values at finite sets, together with the fact that the value of a $J$-term at each 
finite set can be calculated, via (4.1), from its values on finite subsets of $I-J$.

7. Properties of $J$-terms. If $t$ is a $J$-term, let $c$ be the $J$-constant obtained by restricting $t$ to the subsets of $I-J$; we shall indicate the dependence of $c$ on $t$ by writing

$$
c=t_{J}
$$

The mapping that sends $t$ onto $t_{J}$ is a one-to-one mapping from all $J$-terms onto all $J$-constants; its inverse is the mapping that sends $c$ onto $\bar{c}$ (cf. (6.3)). In other words, if $t$ is a $J$-term, and if $c=t_{J}$, then $t=\bar{c}$; similarly, if $c$ is a $J$ constant, and if $t=\bar{c}$, then $c=t_{J}$. All the properties of $J$-terms can be studied by means of this correspondence between $J$-terms and $J$-constants.

(7.2) Lemma. If $t$ is a J-term, then $\mathbf{S}(\varnothing / t)$ is the identity mapping on $\boldsymbol{A}$.

Proof. By (T1), $\mathbf{S}\left(\varnothing / t_{J}\right) p=p$ for all $p$; the assertion follows from the fact that if $K \subset I-J$ (and, in particular, if $K=\varnothing$ ), then $\mathbf{S}(K / t)=\mathbf{S}\left(K / t_{J}\right)$.

(7.3) Lemma. If $t$ is a J-term and if $K \subset I$, then

$$
\mathbf{S}(K / t)=\mathbf{S}(K-J / t) \mathbf{S}(K \cap J / t) .
$$

Proof. Suppose first that $K$ is finite. Let $p$ be an element of $A$ with finite support $L$ and let $\tau$ be a transformation of type $(K, L \cup J)$. If $\rho=\tau$ on $K-J$ and $\rho=\delta$ outside $K-J$, and if $\sigma=\tau$ on $K \cap J$ and $\sigma=\delta$ outside $K \cap J$, then the transformations $\rho$ and $\sigma$ are of types $(K-J, L \cup J)$ and $(K \cap J, L \cup J)$ respectively, and $\rho \sigma=\tau$. By (5.3), $J \cup L$ supports $q=\mathbf{S}(\sigma(K \cap J) / t) \mathbf{S}(\sigma) p$; it follows that

$$
\begin{aligned}
\mathbf{S}(K-J / t) \mathbf{S}(K \cap J / t) p & =\mathbf{S}(K-J / t) q \\
& =\mathbf{S}(\rho(K-J) / t) \mathbf{S}(\rho) \mathbf{S}(\sigma(K \cap J) / t) \mathbf{S}(\sigma) p \\
& =\mathbf{S}(\rho(K-J) / t) \mathbf{S}(\sigma(K \cap J) / t) \mathbf{S}(\rho) \mathbf{S}(\sigma) p[\text { by (T5)] } \\
& =\mathbf{S}(\tau(K-J) / t) \mathbf{S}(\tau(K \cap J) / t) \mathbf{S}(\tau) p \\
& =\mathbf{S}(\tau K / t) \mathbf{S}(\tau) p[\text { by (T2)] }=\mathbf{S}(K / t) p .
\end{aligned}
$$

In the general case, let $u$ be the mapping from subsets $K$ of $I$ into operators $\mathbf{S}(K / u)$ on $\boldsymbol{A}$ defined by

$$
\mathbf{S}(K / u)=\mathbf{S}(K-J / t) \mathbf{S}(K \cap J / t) .
$$

Clearly (i) $\mathbf{S}(K / u)$ is a Boolean endomorphism of $\boldsymbol{A}$ for each subset $K$ of $I$. If $K \subset I-J$, then

$$
\mathbf{S}(K / u)=\mathbf{S}(K / t) \mathbf{S}(\varnothing / t)=\mathbf{S}(K / t)[\text { by (7.2)]; }
$$

this implies that (ii) the restriction of $u$ to the subsets of $I-J$ is a $J$-constant (namely $t_{J}$ ). Finally (iii) if $p$ is an element of $A$ with finite support $L$, if $K$ is a finite subset of $I$, and if $\sigma$ is a transformation of type $(K, L \cup J)$, then 


$$
\begin{aligned}
\mathbf{S}(K / u) p & =\mathbf{S}(K-J / t) \mathbf{S}(K \cap J / t)=\mathbf{S}(K / t) \text { [by the preceding paragraph] } \\
& =\mathbf{S}(\sigma K / t) \mathbf{S}(\sigma) p=\mathbf{S}(\sigma K-J / t) \mathbf{S}(\sigma K \cap J / t) \mathbf{S}(\sigma) p \\
& =\mathbf{S}(\sigma K / u) \mathbf{S}(\sigma) p
\end{aligned}
$$

The assertions (i), (ii), and (iii) add up to the fact that $u$ is a $J$-term; since $u_{J}=t_{J}$, it follows that $u=t$.

(7.4) Lemma. If $t$ is a $J$-term and if $H$ and $K$ are subsets of $I$, then

$$
\mathbf{S}(H \cup K / t)=\mathbf{S}(H-J / t) \mathbf{S}(K-J / t) \mathbf{S}((H \cup K) \cap J / t) .
$$

Proof. By (7.3)

$$
\mathbf{S}(H \cup K / t)=\mathbf{S}((H \cup K)-J / t) \mathbf{S}((H \cup K) \cap J / t) ;
$$

the conclusion follows from (T2).

(7.5). LEMмA. If $t$ is a $J$-term, if $L$ supports $p$, and if $K \subset I$, then $J \cup(L-K)$ supports $\mathbf{S}(K / t) p$.

Proof. Let $L_{0}$ be a finite support of $p$ such that $L_{0} \subset L$, and let $\sigma$ be a transformation of type $(K \cap J, L \cup J)$. Since, by (7.3),

$$
\mathbf{S}(K / t) p=\mathbf{S}(K-J / t) \mathbf{S}(K \cap J / t) p=\mathbf{S}(K-J / t) \mathbf{S}(\sigma(K \cap J) / t) \mathbf{S}(\sigma) p,
$$

and since, by (5.3), the set

$$
J \mathbf{u}\left(L_{0}-(K \cap J)\right)\left(=J \mathbf{u}\left(L_{0}-K\right) \mathbf{u}\left(L_{0}-J\right)\right)
$$

supports $\mathbf{S}(\sigma(K \cap J) / t) \mathbf{S}(\sigma) p$, it follows, again by (5.3), that the set

$$
J \mathbf{u}\left[\left(\left(L_{0}-K\right) \mathbf{u}\left(L_{0}-J\right)\right)-(K-J)\right]
$$

supports $\mathbf{S}(K / t) p$. The conclusion follows from the fact that

$$
\left(\left(L_{0}-K\right) \mathbf{v}\left(L_{0}-J\right)\right)-(K-J) \subset L_{0}-K \subset L-K .
$$

(7.6) Lemma. If $t$ is a $J$-term, if $L$ supports $p$, and if $K \subset I$, then

$$
\mathbf{S}(K / t) p=\mathbf{S}(K \cap L / t) p .
$$

Proof. Suppose first that $K \subset J$. Let $L_{0}$ be a finite support of $p$ such that $L_{0} \subset L$ and write $L_{1}=L_{0} \cup(K \cap L)$. It follows that $L_{1}$ is a finite support of $p$ and that $K \cap L_{1}=K \cap L$. Consequently it is sufficient, in this case, to prove the conclusion under the added assumption that $L$ is finite. We make this assumption. Let $\sigma$ be a transformation of type $(K, L \cup J)$; write $\tau=\sigma$ on $K \cap L$ and $\tau=\delta$ outside $K \cap L$. It follows that $\tau$ is a transformation of type $(K \cap L, L \cup J)$, and hence that

$$
\mathbf{S}(K \cap L / t) p=\mathbf{S}(\tau(K \cap L) / t) \mathrm{S}(\tau) p .
$$

We have, by (T2), 


$$
\mathbf{S}(K / t) p=\mathbf{S}(\sigma K / t) \mathbf{S}(\sigma) p=\mathbf{S}(\sigma(K \cap L) / t) \mathbf{S}(\sigma(K-L) / t) \mathbf{S}(\sigma) p .
$$

Since $\sigma L$ supports $\mathbf{S}(\sigma) p$ and is disjoint from $\sigma(K-L)$, we have also

$$
\begin{aligned}
\mathbf{S}(\sigma(K-L) / t) \mathbf{S}(\sigma) p & =\mathbf{S}(\sigma(K-L) / t) \exists(I-\sigma L) \mathbf{S}(\sigma) p \\
& =\mathbf{S}(\sigma(K-L) / t) \exists((I-J)-\sigma L) \exists(J \cap \sigma L) \mathbf{S}(\sigma) p \\
& =\exists((I-J)-\sigma L) \exists(J \mathbf{n} \sigma L) \mathbf{S}(\sigma) p[\mathrm{by}(\mathrm{T} 3) \text { and (T1) }] \\
& =\exists(I-\sigma L) \mathbf{S}(\sigma) p=\mathbf{S}(\sigma) p .
\end{aligned}
$$

The preceding three equations together imply that

$$
\begin{aligned}
\mathbf{S}(K / t) p & =\mathbf{S}(\sigma(K \cap L) / t) \mathbf{S}(\sigma) p \\
& =\mathbf{S}(\tau(K \cap L) / t) \mathbf{S}(\tau) p[\text { since } \tau=\sigma \text { on } L] \\
& =\mathbf{S}(K \cap L / t) p .
\end{aligned}
$$

In the general case

$$
\begin{aligned}
\mathbf{S}(K / t) p & =\mathbf{S}(K-J / t) \mathbf{S}(K \cap J / t) p[\text { by (7.3)] } \\
& =\mathbf{S}(K-J / t) \mathbf{S}(K \cap J \cap L / t) p \text { [by the preceding paragraph] } \\
& =\mathbf{S}((K-J) \cap L / t) \mathbf{S}(K \cap J \cap L / t) p[\text { by (5.1)] } \\
& =\mathbf{S}(K \cap L / t) p[\text { by (7.3)]; }
\end{aligned}
$$

this completes the proof of Lemma (7.6).

(7.7) Lemma. If $t$ is a $J$-term and if $H$ and $K$ are subsets of $I$, then

$$
\mathbf{S}(H / t) \exists(K)=\exists(K-J) \mathbf{S}(H-K / t) \exists(K \cap J) .
$$

Proof. We shall first prove the special case of the lemma in which $H \subset J$ and $K$ is a finite subset of $I-J$; in that case the desired conclusion takes the form

$$
\mathbf{S}(H / t) \exists(K)=\exists(K) \mathbf{S}(H / t) .
$$

Let $p$ be an element of $\boldsymbol{A}$ with finite support $L$, and let $\sigma$ be a transformation of type $(H, L \cup J \cup K)$. It follows that $\sigma$ is of type $(H, L \cup J)$, and therefore

$$
\mathbf{S}(H / t) p=\mathbf{S}(\sigma H / t) \mathbf{S}(\sigma) p
$$

and

$$
\mathbf{S}(H / t) \exists(K) p=\mathbf{S}(\sigma H / t) \mathbf{S}(\sigma) \exists(K) p .
$$

Since $K \cap L \subset K \subset I-J$, and since $\sigma H$ is disjoint from $K$, it follows that $\sigma$ lives outside $K \cap L$, and hence that

$$
\mathbf{S}(\sigma) \exists(K \cap L)=\exists(K \cap L) \mathbf{S}(\sigma) .
$$

The same reasons imply, via (T3), that 


$$
\mathbf{S}(\sigma H / t) \exists(K \cap L)=\exists(K \cap L) \mathbf{S}(\sigma H / t) .
$$

It follows, since $\exists(K) p=\exists(K \cap L) p$, that

$$
\mathbf{S}(H / t) \exists(K) p=\exists(K \cap L) \mathbf{S}(\sigma H / t) \mathbf{S}(\sigma) p .
$$

Since $J \cup L$ supports $\mathbf{S}(H / t) p$ and since $K-L$ is disjoint from $J \cup L$, it follows that $\exists(K-L)$ leaves $\mathbf{S}(H / t) p$ invariant, and hence that

$$
\mathbf{S}(H / t) \exists(K) p=\exists(K) \mathbf{S}(\sigma H / t) \mathbf{S}(\sigma) p=\exists(K) \mathbf{S}(H / t) p,
$$

as desired.

Suppose next that $K$ is an arbitrary (not necessarily finite) subset of $I-J$. If $p$ is an element of $A$ with finite support $L$, and if $K_{0}=K \cap L$, then

$$
\begin{aligned}
\mathbf{S}(H / t) \exists(K) p & =\mathbf{S}(H / t) \exists(K \cap L) p=\mathbf{S}(H / t) \exists\left(K_{0}\right) p \\
& =\exists\left(K_{0}\right) \mathbf{S}(H / t) p[\text { by }(7.8)]=\exists(K \cap L) \mathbf{S}(H / t) p .
\end{aligned}
$$

(We are still assuming that $H \subset J$.) Since $J \cup L$ supports $\mathbf{S}(H / t) p$ and is disjoint from $K-L$, it follows that $\exists(K-L)$ leaves $\mathbf{S}(H / t) p$ invariant; this, together with the preceding equation, implies that

$$
\mathbf{S}(H / t) \exists(K)=\exists(K) \mathbf{S}(H / t)
$$

whenever $H \subset J$ and $K \subset I-J$.

We assert next that if $H$ and $K$ are quite arbitrary subsets of $I$, then

$$
\mathbf{S}(H / t) \exists(K)=\exists(K-J) \mathbf{S}(H / t) \exists(K \cap J) .
$$

Indeed :

$$
\begin{aligned}
& \mathbf{S}(H / t) \exists(K)=\mathbf{S}(H-J / t) \mathbf{S}(H \cap J / t) \exists(K-J) \exists(K \cap J) \text { [by (7.3)] } \\
& =\mathbf{S}(H-J / t) \exists(K-J) \mathbf{S}(H \cap J / t) \exists(K \cap J) \text { [by (7.9)] } \\
& =\exists(K-J) \mathbf{S}(H-J / t) \mathbf{S}(H \cap J / t) \exists(K \cap J) \text { [by (T3)] } \\
& =\exists(K-J) \mathbf{S}(H / t) \exists(K \cap J) \text { [by (7.3)]. }
\end{aligned}
$$

Replacing $H$ by $H-K$ in (7.10) we obtain

$$
\mathbf{S}(H-K / t) \exists(K)=\exists(K-J) \mathbf{S}(H-K / t) \exists(K \cap J) .
$$

Since $I-K$ supports $\exists(K) p$ for every $p$ in $A$, it follows that

$$
\mathbf{S}(H / t) \exists(K) p=\mathbf{S}(H-K / t) \exists(K) p[\text { by }(7.6)] ;
$$

(7.10), (7.12), and (7.11) imply the desired conclusion.

(7.13) Lemma. If $t$ is a J-term and if $H$ and $K$ are subsets of $I$, then

$$
\exists(H) \mathbf{S}(K / t)=\exists(H \cap J) \mathbf{S}(K / t) \exists(H-(J \cup K)) .
$$

Proof. Compute, as follows: 


$$
\begin{aligned}
\exists(H) \mathbf{S}(K / t) & =\exists(H \cap J) \exists(H-J) \mathbf{S}(K-J / t) \mathbf{S}(K \cap J / t) \text { [by (7.3)] } \\
& =\exists(H \cap J) \mathbf{S}(K-J / t) \exists((H-J)-(K-J)) \mathbf{S}(K \cap J / t)[\text { by (T4)] } \\
& =\exists(H \cap J) \mathbf{S}(K-J / t) \exists(H-(J \cup K)) \mathbf{S}(K \cap J / t) \\
& =\exists(H \cap J) \mathbf{S}(K-J / t) \mathbf{S}(K \cap J / t) \exists(H-(J \cup K))[\text { by (7.8)] } \\
& =\exists(H \cap J) \mathbf{S}(K / t) \exists(H-(J \cup K))[\text { by (7.3)]. }
\end{aligned}
$$

8. Terms and transformations. In the preceding section we discussed what might be called the commutation relations involving terms and the quantifier structure of $A$; in this section we do the same thing for the transformation structure of $A$.

It is convenient to begin by introducing a new item of notation and making one or two elementary remarks on it. If $\tau$ is a transformation on $I$ and if $J$ is a subset of $I$, we shall denote by $\tau_{J}$ the transformation such that

$$
\tau_{J}=\tau \text { on } \tau^{-1} J \text { and } \tau_{J}=\delta \text { outside } \tau^{-1} J \text {. }
$$

Observe that if $\tau$ happens to live on $J$, then $\tau^{-1} J=J$, and therefore $\tau_{J}=\tau$ and $\tau_{I-J}=\delta$; if, on the other hand, $\tau$ lives on $I-J$, then $\tau_{J}=\delta$ and $\tau_{I-J}=\tau$.

(8.1) Lemma. If $\tau=\delta$ on $J$, then $\tau_{I-J}$ lives on $I-J$ and $\tau_{I-J} \tau_{J}=\tau$.

Proof. If $i \epsilon J$, then $\tau i=i \epsilon J$, and therefore $\tau_{I-\tau} i=i$. If $i \epsilon^{\prime} J$, then either $\tau i \epsilon J$, in which case $\tau_{I-J} i=i \epsilon^{\prime} J$, or $\tau i \epsilon^{\prime} J$, in which case $\tau_{I-J} i=\tau i \epsilon^{\prime} J$. This proves that $\tau_{I-J}$ lives on $I-J$. To prove the asserted equation, observe that if $i \epsilon \tau^{-1} J$, then (since $\tau=\delta$ on $J$ ) $\tau \tau i=\tau i \epsilon J$ and therefore $\tau_{I-J} \tau_{J} i=\tau_{I-J} \tau i=\tau i$, while if $i \epsilon^{\prime} \tau^{-1} J$, then $\tau_{I-J} \tau_{J} i=\tau_{I-J} i=\tau i$.

(8.2) Lemma. If $t$ is a $J$-term, if $\tau$ is a transformation such that $\tau=\delta$ on $J$, and if $K \subset I$, then

$$
\mathbf{S}(K / t) \mathbf{S}(\tau)=\mathbf{S}\left(\tau_{I-J}\right) \mathbf{S}\left(\left(\tau_{I-I}\right)^{-1} K / t\right) \mathbf{S}\left(\tau_{J}\right) .
$$

Proof. Suppose first that the transformation $\tau$ is finite, and let $H$ be a finite set such that $\tau$ lives on $H$. Given an element $p$ of $A$, let $L$ be a finite support of $p$. If $\sigma$ is a transformation of type $(K \cap J, L \cup J \cup H)$, then, since $\tau L$ supports $\mathbf{S}(\tau) p$ and since $\tau L \subset L \cup J \cup H$, it follows (via (7.3)) that

$$
\mathbf{S}(K / t) \mathbf{S}(\tau) p=\mathbf{S}(K-J / t) \mathbf{S}(\sigma(K \cap J) / t) \mathbf{S}(\sigma) \mathbf{S}(\tau) p .
$$

Since $\tau_{I-J} i$ is always equal either to $\tau i$ or to $i$, it follows that $\tau_{I-J}$ lives on $H$ (along with $\tau$ ), and hence (by (8.1)) that it lives on $H \cap(I-J)=H-J$. Since $\sigma$ lives on $(K \cap J) \cup \sigma(K \cap J)$, and since that set is disjoint from $H-J$, it follows that $\sigma$ commutes with $\tau_{I-J}$. Consequently (via another use of (8.1))

$$
\begin{aligned}
\mathbf{S}(K / t) \mathbf{S}(\tau) p & =\mathbf{S}(K-J / t) \mathbf{S}(\sigma(K \cap J) / t) \mathbf{S}\left(\tau_{I-J}\right) \mathbf{S}(\sigma) \mathbf{S}\left(\tau_{J}\right) p \\
& =\mathbf{S}(K-J / t) \mathbf{S}\left(\tau_{I-J}\right) \mathbf{S}\left(\left(\tau_{I-J}\right)^{-1} \sigma(K \cap J) / t\right) \mathbf{S}(\sigma) \mathbf{S}\left(\tau_{J}\right) p .
\end{aligned}
$$

Since $\tau_{I-J}$ lives on $I-J$ and since $K-J \subset I-J$, it follows that $\left(\tau_{I-J}\right)^{-1}(K-J)$ 
$\subset I-J$. Since $\tau_{I-J}$ lives on $H$ and since $\sigma(K \cap J) \subset I-H$, it follows that $\left(\tau_{I-J}\right)^{-1} \sigma(K \cap J)=\sigma(K \cap J)$. These two remarks and (8.3) imply that

$$
\mathbf{S}(K / t) \mathbf{S}(\tau) p=\mathbf{S}\left(\tau_{I-J}\right) \mathbf{S}\left(\left(\tau_{I-J}\right)^{-1}(K-J) / t\right) \mathbf{S}(\sigma(K \cap J) / t) \mathbf{S}(\sigma) \mathbf{S}\left(\tau_{J}\right) p .
$$

Observe next that (since $\tau=\delta$ on $J$ )

$$
\left(\tau_{I-J}\right)^{-1} K-J=\left(\tau_{I-J}\right)^{-1}(K-J) \text { and }\left(\tau_{I-J}\right)^{-1} K \cap J=K \cap J .
$$

Since $\tau_{J} L$ supports $\mathbf{S}\left(\tau_{J}\right) p$ and $\tau_{J} L \subset L \cup J \cup H$, it follows from (8.4) that

$$
\mathbf{S}(K / t) \mathbf{S}(\tau) p=\mathbf{S}\left(\tau_{I-J}\right) \mathbf{S}\left(\left(\tau_{I-J}\right)^{-1} K-J / t\right) \mathbf{S}\left(\left(\tau_{I-J}\right)^{-1} K \cap J / t\right) \mathbf{S}\left(\tau_{J}\right) p ;
$$

the proof (in the case that $\tau$ is finite) is complete.

Suppose next that $\tau$ lives on $I-J$ and that $K \subset J ;$ i.: this case the desideratum takes the form

$$
\mathbf{S}(K / t) \mathbf{S}(\tau)=\mathbf{S}(\tau) \mathbf{S}\left(\tau^{-1} K / t\right) .
$$

Given an element $p$ of $A$, let $L$ be a finite support of $p$; write $\rho=\tau$ on $L$ and $\rho=\delta$ outside $L$. The transformation $\rho$ is finite and $\mathbf{S}(\tau) p=\mathbf{S}(\rho) p$. Since $\rho$ lives on $I-J$ (along with $\tau$ ), it follows that $\rho_{I-J}=\rho$ and $\rho_{J}=\delta$; the preceding paragraph implies therefore that

$$
\mathbf{S}(K / t) \mathbf{S}(\tau) p=\mathbf{S}(K / t) \mathbf{S}(\rho) p=\mathbf{S}(\rho) \mathbf{S}\left(\rho^{-1} K / t\right) p .
$$

Since $L \cup J$ supports $\mathbf{S}\left(\rho^{-1} K / t\right) p$ and since $\rho=\tau$ on $L \cup J$, it follows that

$$
\mathbf{S}(K / t) \mathbf{S}(\tau) p=\mathbf{S}(\tau) \mathbf{S}\left(\rho^{-1} K / t\right) p .
$$

By (7.6),

$$
\mathbf{S}\left(\tau^{-1} K / t\right) p=\mathbf{S}\left(\tau^{-1} K \cap L / t\right) p \quad \text { and } \quad \mathbf{S}\left(\rho^{-1} K / t\right) p=\mathbf{S}\left(\rho^{-1} K \cap L / t\right) p .
$$

Now $\tau^{-1} K \cap L=\rho^{-1} K \cap L$ (by the definition of $\rho$ ), and the proof of (8.5) is complete.

The general case is deduced from the special case just treated, as follows:

$$
\begin{aligned}
\mathbf{S}(K / t) \mathbf{S}(\tau) & =\mathbf{S}(K-J / t) \mathbf{S}(K \cap J / t) \mathbf{S}\left(\tau_{I-J}\right) \mathbf{S}\left(\tau_{J}\right) \text { [by (7.3) and (8.1)] } \\
& =\mathbf{S}(K-J / t) \mathbf{S}\left(\tau_{I-J}\right) \mathbf{S}\left(\left(\tau_{I-J}\right)^{-1}(K \cap J) / t\right) \mathbf{S}\left(\tau_{J}\right)[\text { by }(8.5)] \\
& =\mathbf{S}\left(\tau_{I-J}\right) \mathbf{S}\left(\left(\tau_{I-J}\right)^{-1}(K-J) / t\right) \mathbf{S}\left(\left(\tau_{I-J}\right)^{-1}(K \cap J) / t\right) \mathbf{S}\left(\tau_{J}\right)[\text { by (T5)] } \\
& =\mathbf{S}\left(\tau_{I-J}\right) \mathbf{S}\left(\left(\tau_{I-J}\right)^{-1} K-J / t\right) \mathbf{S}\left(\left(\tau_{I-J}\right)^{-1} K \cap J / t\right) \mathbf{S}\left(\tau_{J}\right)[\text { by (8.1)] } \\
& =\mathbf{S}\left(\tau_{I-J}\right) \mathbf{S}\left(\left(\tau_{I-J}\right)^{-1} K / t\right) \mathbf{S}\left(\tau_{J}\right)[\text { by }(7.3)] .
\end{aligned}
$$

This concludes the proof of (8.2).

(8.6) LeMma. If $t$ is a $J$-term, if $\tau$ is a transformation such that $\tau=\delta$ on $J$ and $\tau=\delta$ outside $\tau^{-1} J$, and if $H \subset I-\tau^{-1} J$, then $\mathbf{S}(H / t)$ and $\mathbf{S}(\tau)$ commute.

Proof. Assume first that the transformation $\tau$ is finite; it follows that the set $\tau^{-1} J-J$ is finite. If that set is empty, then $\tau=\delta$ and the conclusion is 
trivial. In the remaining case, let $j_{1}, \cdots, j_{n}$ be the distinct elements of $\tau^{-1} J-J$; it is easy to verify that

$$
\tau=\left(j_{1} / \tau j_{1}\right) \cdots\left(j_{n} / \tau j_{n}\right),
$$

and that the factors of this product commute among themselves. Since the sets $\left\{j_{1}\right\}, \cdots,\left\{j_{n}\right\}$, and $H$ are pairwise disjoint, it follows from the commutativity theorem (1.3) for constants (applied to the $J$-constants induced by the variables $\tau j_{1}, \cdots, \tau j_{n}$ and by the $J$-term $t$ ) that the Boolean endomorphisms $\mathbf{S}\left(j_{1} / \tau j_{1}\right), \cdots, \mathbf{S}\left(j_{n} / \tau j_{n}\right)$, and $\mathbf{S}(H / t)$ commute among themselves. Since

$$
\mathbf{S}(\tau)=\mathbf{S}\left(j_{1} / \tau j_{1}\right) \cdots \mathbf{S}\left(j_{n} / \tau j_{n}\right),
$$

the desideratum is proved in this case.

In the general case we argue separately for each element of $\boldsymbol{A}$. Given $p$ in $A$, let $L$ be a finite support of $p$; write $\tau_{0}=\tau$ on $L$ and $\tau_{0}=\delta$ outside $L$. Since $\tau_{0}=\delta$ whenever $\tau=\delta$, it follows that $\tau_{0}=\tau$ on $J \cup L$, and hence that $\tau_{0}=\tau$ on a support of $\mathbf{S}(H / t) p$. The preceding paragraph implies that

$$
\mathbf{S}(H / t) \mathbf{S}(\tau) p=\mathbf{S}(H / t) \mathbf{S}\left(\tau_{0}\right) p=\mathbf{S}\left(\tau_{0}\right) \mathbf{S}(H / t) p=\mathbf{S}(\tau) \mathbf{S}(H / t) p ;
$$

this completes the proof of the lemma.

(8.7) Lemma. If $t$ is a $J$-term, if $\tau$ is a transformation such that $\tau=\delta$ on $J$, and if $K \subset I-\tau^{-1} J$, then

$$
\mathbf{S}(K / t) \mathbf{S}(\tau)=\mathbf{S}(\tau) \mathbf{S}\left(\tau^{-1} K / t\right) .
$$

Proof. The assumptions on $\tau$ and $K$ imply that

$$
\left(\tau_{I-J}\right)^{-1} K=\tau^{-1} K \text {. }
$$

Indeed, if $\tau i \in K$ (i.e., $i \in \tau^{-1} K$ ), then $i \in \tau^{-1}(I-J)$, so that $\tau_{I-J} i=\tau i$, and therefore $\tau_{I-J} i \in K$. If, on the other hand, $\tau_{I-J} i \epsilon K$, then $\tau i \epsilon I-J$ (i.e., $\left.i \epsilon \tau^{-1}(I-J)\right)$. The reason is that if $\tau i \epsilon^{\prime} I-J$, then $\tau_{I-J} i=i$, so that $i \epsilon K$ and $\tau i \in J$; this contradicts the fact that $K \cap \tau^{-1} J=\varnothing$. It follows that $\tau_{I-J} i=\tau i$, and, therefore, that $\tau i \in K$.

By (8.2) and (8.8),

$$
\mathbf{S}(K / t) \mathbf{S}(\tau)=\mathbf{S}\left(\tau_{I-J}\right) \mathbf{S}\left(\tau^{-1} K / t\right) \mathbf{S}\left(\tau_{J}\right) .
$$

Since $K \subset I-\tau^{-1} J \subset I-J$, it follows that $\tau^{-1} K \subset I-\tau^{-1} J$, so that (8.6) is applicable to $\tau_{J}$ and $\tau^{-1} K$ in place of $\tau$ and $H$, respectively. The desired conclusion follows from (8.9) and (8.1).

For convenience of reference we proceed to summarize the most important ones among the results about $J$-terms obtained above.

(8.10) THEOREM. If $t$ is a J-term, if $H$ and $K$ are subsets of $I$, and if $\tau$ is a transformation such that $\tau=\delta$ on $J$, then 
(T) $\quad \mathbf{S}(\varnothing / t)$ is the identity mapping on $\boldsymbol{A}$,

$(\overline{\mathrm{T}} 2) \quad \mathbf{S}(H \cup K / t)=\mathbf{S}(H-J / t) \mathbf{S}(K-J / t) \mathbf{S}((H \cup K) \cap J / t)$,

(页3) $\mathbf{S}(H / t) \exists(K)=\exists(K-J) \mathbf{S}(H-K / t) \exists(K \cap J)$,

$(\overline{\mathrm{T}} 4) \quad \exists(H) \mathbf{S}(K / t)=\exists(H \cap J) \mathbf{S}(K / t) \exists(H-(J \cap K))$,

(宁5) $\quad \mathbf{S}(K / t) \mathbf{S}(\tau)=\mathbf{S}\left(\tau_{I-J}\right) \mathbf{S}\left(\left(\tau_{I-J}\right)^{-1} K / t\right) \mathbf{S}\left(\tau_{J}\right)$.

We conclude this section by a brief description of how its results are connected with the corresponding results for $J$-constants. In view of the extension theorem (6.7), ( $\overline{\mathrm{T}} 1)$ says the same thing as (T1). (The extension theorem is frequently used, above and below, without explicit reference.) If $H$ and $K$ are subsets of $I-J$, ( $\overline{\mathrm{T}} 2)$ reduces to (T2). If $K \subset I-J$, then ( $\overline{\mathrm{T}} 3$ ) reduces to a generalization of (T3); the assumption $H \subset I-J$ is not necessary. If $H \subset I-J$, then $(\overline{\mathrm{T}} 4)$ reduces to a generalization of (T4); the assumption $K \subset I-J$ is not necessary. If $\tau$ lives on $I-J$, then ( $\overline{\mathrm{T}} 5)$ reduces to a generalization of (T5); the assumption $K \subset I-J$ is not necessary. The assertion (8.7) is also a generalization of (T5), in a different direction. It asserts that if $K$ is suitably specialized, then it is not necessary to assume that $\tau$ lives on $I-J$; it is sufficient to assume merely that $\tau=\delta$ on $J$.

9. The construction of terms. To construct terms, it is useful to know that a term, like a constant, is uniquely determined by a single Boolean endomorphism.

(9.1) LeммA. If $J$ is a finite subset of $I$, if $f$ is a Boolean endomorphism of $A$, and if $i$ is an element of $I-J$ such that

$$
\begin{aligned}
& \exists(i) f=f, \\
& f \exists(i)=\exists(i), \\
& f \exists(j)=\exists(j) f \text { whenever } j \neq i \text { and } j \in I-J,
\end{aligned}
$$

then there exists a unique $J$-term $t$ of $\boldsymbol{A}$ such that $\mathbf{S}(i / t)=f$.

This lemma is an immediate consequence of the corresponding result (1.2) for constants (applied to the algebra obtained from $\boldsymbol{A}$ by fixing the variables of $J$ ) together with the theorem (6.7) on the extension of $J$-constants to $J$ terms.

In the applications it often happens that it is easy to define a Boolean homomorphism $g$ from a part of $\boldsymbol{A}$ into $\boldsymbol{A}$ so that $g$ satisfies the conditions of (9.1) as far as the limitations of its domain allow. The domains that arise are "finite compressions" of $A$, i.e., they are obtained by taking a finite subset $K$ of $I$ and considering only those elements of $A$ that are independent of $K$. In other words, such a domain consists of all those elements $p$ of $\boldsymbol{A}$ for which $\exists(K) p=p$, or, equivalently, it is the range of the quantifier $\exists(K)$. Our next result says that under a relatively mild additional restriction (involving the transformation structure of $\boldsymbol{A}$ ) even such a partial endomorphism yields 
a term. The result is a generalization of $(9.1)$; the proof is based on an application of that lemma. To prepare the ground we apply a slight refinement of the techniques that were used to extend $J$-constants to $J$-terms. The idea of the proof is to extend the given partial endomorphism $g$ to an honest endomorphism $f$ to which (9.1) applies. To do this, we first transform the variables of each element of $\boldsymbol{A}$ so as to obtain an element in the domain of $g$, then we apply $g$, and then, finally, we undo the effect of the first transformation. All this is, of course, a vague qualitative description; we proceed now to the precise details.

(9.2) THEOREM. If $J$ and $K$ are finite subsets of $I$, if $g$ is a Boolean homomorphism from the range of $\exists(K)$ into $A$, and if $i$ is an element of $I-(J \cup K)$ such that

(1) $\exists(i) g \exists(K)=g \exists(K)$,

(2) $g \exists(K) \exists(i)=\exists(K) \exists(i)$,

(3) $g \exists(K) \exists(j)=\exists(j) g \exists(K)$ whenever $j \neq i$ and $j \in I-J$,

(4) $g \exists(K) \mathbf{S}(\tau)=\mathbf{S}(\tau) g \exists(K)$ whenever $\tau$ is a finite transformation that lives on $I-(\{i\} \cup J \cup K)$,

then there exists a J-term $t$ of $\boldsymbol{A}$ such that $\mathbf{S}(i / t) \exists(K)=g \exists(K)$.

Remark. If $K=\varnothing$, then (1), (2), and (3) coincide with the corresponding conditions of (9.1), and therefore, in that case, (4) is superfluous. In the general case it is an immediate consequence of (T5) that the condition (4) is necessary for the existence of $t$.

Proof. Given an element $p$ of $\boldsymbol{A}$ with finite support $L$, let $\sigma$ be a transformation of type $(K,\{i\} \cup L \cup J)$, and define an operator $f$ on $\boldsymbol{A}$ by writing

$$
f p=\mathbf{S}(\tilde{\boldsymbol{\sigma}}) g \mathbf{S}(\sigma) p .
$$

(For the definition of $\tilde{\sigma}$, see (3.2).) We observe that since $\sigma L$ supports $\mathbf{S}(\sigma) p$ and is disjoint from $K$, the element $\mathbf{S}(\sigma) p$ is independent of $K$ and, consequently, belongs to the domain of $g$. Thus the definition (9.3) makes sense, but it must, of course, be supported by an unambiguity proof.

If both $\sigma$ and $\tau$ are transformations of type $(K,\{i\} \cup L \cup J)$, apply (3.4) to find a permutation $\pi$ such that $\pi \tau=\sigma \pi, \pi \tilde{\tau}=\tilde{\sigma} \pi$, and $\pi=\delta$ in $\{i\} \cup J \cup K$ u $L$. Form $\mathbf{S}(\tilde{\tau}) g \mathbf{S}(\tau) p$, and then replace $\mathbf{S}(\tau)$ by $\mathbf{S}\left(\pi^{-1}\right) \mathbf{S}(\sigma) \mathbf{S}(\pi)$ and $\mathbf{S}(\tilde{\tau})$ by $\mathbf{S}\left(\pi^{-1}\right) \mathbf{S}(\tilde{\sigma}) \mathbf{S}(\pi)$. Since $\pi=\delta$ in $L$ and since $L$ supports $p$, the rightmost $\mathbf{S}(\pi)$ may be erased from the resulting equation. Since $\pi$ lies on $I-(\{i\} \cup J \cup K)$, it follows from (9.2)(4) that the rightmost $\mathbf{S}\left(\pi^{-1}\right)$ can be pulled to the left of $g$. As a consequence of these maripulations we obtain

$$
\mathbf{S}(\tilde{\tau}) g \mathbf{S}(\tau) p=\mathbf{S}\left(\pi^{-1}\right) \mathbf{S}(\tilde{\sigma}) g \mathbf{S}(\sigma) p .
$$

In order to prove now that the factor $\mathbf{S}\left(\pi^{-1}\right)$ can be erased from (9.4), we 
investigate the supports of some of the constituents of that equation. We know that $\sigma L$ supports $\mathbf{S}(\sigma) p$; we assert that

$$
\sigma L \cup J \text { supports } g \mathbf{S}(\sigma) p \text {. }
$$

If $j \epsilon^{\prime} \sigma L \cup J$, then either $j \neq i$, in which case

$$
\exists(j) g \mathbf{S}(\sigma) p=g \exists(j) \mathbf{S}(\sigma) p[\text { by }(9.2)(3)]=g \mathbf{S}(\sigma) p\left[\text { since } j \epsilon^{\prime} \sigma L\right],
$$

or $j=i$, in which case

$$
\exists(j) g \mathbf{S}(\sigma) p=\exists(i) g \mathbf{S}(\sigma) p=g \mathbf{S}(\sigma) p[\text { by (9.2)(1)]. }
$$

In any case, therefore, if $j \epsilon^{\prime} \sigma L \cup J$, then $g \mathbf{S}(\sigma) p$ is independent of $j$; this proves (9.5). Since

$$
\tilde{\sigma}(\sigma L \cup J)=\tilde{\sigma} L \mathbf{u} J=L \mathbf{u} J
$$

it follows that

$$
L \cup J \text { supports } \mathbf{S}(\tilde{\sigma}) g \mathbf{S}(\sigma) p .
$$

Since $\pi^{-1}=\delta$ on $L \cup J$, it follows from (9.4) and (9.6) that

$$
\mathbf{S}(\tilde{\tau}) g \mathbf{S}(\tau) p=\mathbf{S}(\tilde{\sigma}) g \mathbf{S}(\sigma) p .
$$

This does not quite finish the unambiguity proof; we must still show that the choice of $L$ is immaterial. For this purpose, suppose that both $L_{1}$ and $L_{2}$ are finite supports of $p$ and that $\sigma_{1}$ and $\sigma_{2}$ are transformations of types $\left(K,\{i\} \cup L_{1} \cup J\right)$ and $\left(K,\{i\} \cup L_{2} \cup J\right)$ respectively. If $L=L_{1} \cup L_{2}$, then $L$ is a finite support of $p$. Let $\tau$ be an arbitrary transformation of type ( $K$, $\{i\} \cup L \cup J)$. Two applications of (9.7), first to $\sigma_{1}$ and $\tau$ and then to $\sigma_{2}$ and $\tau$, imply that

$$
\mathbf{S}\left(\tilde{\sigma}_{1}\right) g \mathbf{S}\left(\sigma_{1}\right) p=\mathbf{S}\left(\tilde{\sigma}_{2}\right) g \mathbf{S}\left(\sigma_{2}\right) p
$$

as desired.

A straightforward argument (cf. (6.4)) shows that the mapping $f$ defined by (9.3) is a Boolean endomorphism of $A$. The next thing to do is to verify that $f$ satisfies the conditions (1), (2) and (3) of (9.1).

With $p, L$, and $\sigma$ as before, we have

$$
\begin{aligned}
\exists(i) f p & =\exists(i) \mathbf{S}(\tilde{\sigma}) g \mathbf{S}(\sigma) p=\mathbf{S}(\tilde{\sigma}) \exists(i) g \mathbf{S}(\sigma) p\left[\text { since } \tilde{\sigma}^{-1}\{i\}=\{i\}\right] \\
& =\mathbf{S}(\tilde{\sigma}) g \mathbf{S}(\sigma) p[\text { by }(9.2)(1)]=f p,
\end{aligned}
$$

and

$$
\begin{aligned}
f \exists(i) p & =\mathbf{S}(\tilde{\sigma}) g \mathbf{S}(\sigma) \exists(i) p=\mathbf{S}(\tilde{\sigma}) g \exists(i) \mathbf{S}(\sigma) p\left[\text { since } \sigma^{-1}\{i\}=\{i\}\right] \\
& =\mathbf{S}(\tilde{\sigma}) \exists(i) \mathbf{S}(\sigma) p[\text { by }(9.2)(2)]=\mathbf{S}(\tilde{\sigma}) \mathbf{S}(\sigma) \exists(i) p \\
& =\mathbf{S}(\tilde{\sigma} \sigma) \exists(i) p=\mathbf{S}(\tilde{\boldsymbol{\sigma}}) \exists(i) p \\
& =\exists(i) p[\text { since } \tilde{\sigma}=\delta \text { outside } \sigma K \text { and } p \text { is independent of } \sigma K]
\end{aligned}
$$


this settles (9.1)(1) and (9.1)(2).

The proof of (9.1)(3) is harder. We continue to use $p, L$, and $\sigma$ as before, and we consider an element $j$ of $I-J$ such that $j \neq i$. If $j \epsilon^{\prime} L$, then $j \epsilon^{\prime} L \mathbf{u} J$ and therefore, since (by (9.6)) $L \cup J$ supports $f p$,

$$
\exists(j) f p=f p \text { and } f \exists(j) p=f p .
$$

If $j \epsilon L-K$, then $\sigma^{-1}\{j\}=\tilde{\sigma}^{-1}\{j\}=\{j\}$ (since $j \epsilon^{\prime} \sigma K$ ), and therefore

$$
\begin{aligned}
\exists(j) f p & =\exists(j) \mathbf{S}(\tilde{\boldsymbol{\sigma}}) g \mathbf{S}(\sigma) p=\mathbf{S}(\tilde{\boldsymbol{\sigma}}) \exists(j) g \mathbf{S}(\sigma) p \\
& =\mathbf{S}(\tilde{\boldsymbol{\sigma}}) g \exists(j) \mathbf{S}(\sigma) p[\mathrm{by}(9.2)(3)]=\mathbf{S}(\tilde{\sigma}) g \mathbf{S}(\sigma) \exists(j) p=f \exists(j) p .
\end{aligned}
$$

The only remaining case is the one in which $j \in L \cap K$.

Let $\sigma_{-}$be the transformation that agrees with $\sigma$ except at $\sigma j$ (where $\sigma=\delta$ ) and that sends $\sigma j$ onto $j$. Since both $p$ and $\exists(j) p$ are independent of $\sigma j$, it follows that

$$
\mathbf{S}(\sigma) p=\mathbf{S}\left(\sigma_{-}\right) p \text { and } \mathbf{S}(\sigma) \exists(j) p=\mathbf{S}\left(\sigma_{-}\right) \exists(j) p .
$$

Note, for later use, that $\sigma_{-}^{-1}\{\sigma j\}=\{j\}$. Let $\sigma_{+}$be the transformation that agrees with $\tilde{\sigma}$ except at $j$ (where $\tilde{\sigma}=\delta$ ) and that sends $j$ onto $\sigma j$. Since both $g \mathbf{S}(\sigma) p$ and $g \mathbf{S}(\sigma) \exists(j) p$ are independent of $j$ (by (9.5)), it follows that

$$
\mathbf{S}(\tilde{\sigma}) g \mathbf{S}(\sigma) p=\mathbf{S}\left(\sigma_{+}\right) g \mathbf{S}(\sigma) p
$$

and

$$
\mathbf{S}(\tilde{\boldsymbol{\sigma}}) g \mathbf{S}(\sigma) \exists(j) p=\mathbf{S}\left(\sigma_{+}\right) g \mathbf{S}(\sigma) \exists(j) p .
$$

The proof of the remaining case of $(9.1)(3)$ is now a matter of computation, as follows:

$$
\begin{aligned}
f \exists(j) p & =\mathbf{S}(\tilde{\sigma}) g \mathbf{S}(\sigma) \exists(j) p=\mathbf{S}\left(\sigma_{+}\right) g \mathbf{S}\left(\sigma_{-}\right) \exists(j) p \\
& =\mathbf{S}\left(\sigma_{+}\right) g \exists(\sigma j) \mathbf{S}\left(\sigma_{-}\right) p=\mathbf{S}\left(\sigma_{+}\right) \exists(\sigma j) g \mathbf{S}\left(\sigma_{-}\right) p \\
& =\exists(j) \mathbf{S}\left(\sigma_{+}\right) g \mathbf{S}\left(\sigma_{-}\right) p=\exists(j) \mathbf{S}(\tilde{\sigma}) g \mathbf{S}(\sigma) p=\exists(j) f p .
\end{aligned}
$$

An application of (9.1) yields the existence of a $J$-term $t$ such that $\mathbf{S}(i / t)=f$. Suppose now that an element $p$ of $\boldsymbol{A}$ is independent of $K$. Let $L$ be a finite support of $p$ such that $L$ is disjoint from $K$, and let $\sigma$ be a transformation of type $(K,\{i\} \cup L \cup J)$. Since $\sigma=\delta$ in $L$, we have $\sigma L=L$ and $\mathbf{S}(\sigma) p=p$. By (9.5), $L \cup J$ supports $g p$; since $\tilde{\sigma}=\delta$ in $L \cup J$, it follows that

$$
\mathbf{S}(i / t) p=f p=\mathbf{S}(\tilde{\boldsymbol{\sigma}}) g \mathbf{S}(\sigma) p=\mathbf{S}(\tilde{\boldsymbol{\sigma}}) g p=g p .
$$

The proof of Theorem (9.2) is complete.

We prove next that the term $t$ of $(9.2)$ is unique.

(9.8) Theorem. If $J$ and $K$ are finite subsets of $I$, if $s$ and $t$ are $J$-terms, and if $i$ is an element of $I-(J \cup K)$ such that 


$$
\mathbf{S}(i / s) \exists(K)=\mathbf{S}(i / t) \exists(K),
$$

then $s=t$.

Proof. We shall show that $\mathbf{S}(i / s)=\mathbf{S}(i / t)$; the desired result will then follow from the uniqueness assertion of (9.1). If $p$ is an element of $A$ with finite support $L$ and if $\sigma$ is a transformation of type $(K,\{i\} \cup L \mathbf{u} J)$, then $\mathbf{S}(\sigma) p$ is independent of $K$; it follows from (9.9) that

$$
\mathbf{S}(i / s) \mathbf{S}(\sigma) p=\mathbf{S}(i / t) \mathbf{S}(\sigma) p
$$

and hence that

$$
\mathbf{S}(\tilde{\sigma}) \mathbf{S}(i / s) \mathbf{S}(\sigma) p=\mathbf{S}(\tilde{\boldsymbol{\sigma}}) \mathbf{S}(i / t) \mathbf{S}(\sigma) p .
$$

By (8.7), first with $s, \tilde{\sigma}$, and $\{i\}$ and then with $t, \tilde{\sigma}$, and $\{i\}$ in the roles of $t, \tau$, and $K$, respectively, $\mathbf{S}(\tilde{\boldsymbol{\sigma}})$ commutes with both $\mathbf{S}(i / s)$ and $\mathbf{S}(i / t)$; since $\tilde{\sigma} \sigma=\tilde{\sigma}$, it follows that

$$
\mathbf{S}(i / s) \mathbf{S}(\tilde{\sigma}) p=\mathbf{S}(i / t) \mathbf{S}(\tilde{\boldsymbol{\sigma}}) p .
$$

Since $\sigma=\delta$ outside $\sigma K$ and since $p$ is independent of $\sigma K$, it follows that $\mathbf{S}(i / s) p=\mathbf{S}(i / t) p$, and the proof of the uniqueness theorem is complete.

10. Terms in predicates. Suppose that $P$ is an $n$-place predicate and that $t_{1}, \cdots, t_{n}$ are terms; the purpose of this section is to show how it is possible to assign a sensible meaning to the expression $P\left(t_{1}, \cdots, t_{n}\right)$.

There is an obvious way to proceed: simply select $n$ variables $i_{1}, \cdots, i_{n}$ and write

$$
P\left(t_{1}, \cdots, t_{n}\right)=\mathbf{S}\left(i_{1} / t_{1}\right) \cdots \mathbf{S}\left(i_{n} / t_{n}\right) P\left(i_{1}, \cdots, i_{n}\right) .
$$

This procedure is open to several objections. The main difficulty is that the choice of the variables $i_{1}, \cdots, i_{n}$ must not be completely arbitrary. If, for instance, they are not all distinct, then the result is not the expected one, not even in the simplest case in which the roles of the terms are played by other variables. (Compare $\mathbf{S}(i / j) \mathbf{S}(i / k) P(i, i)$ with $P(j, k)$.) If the auxiliary variables are distinct, there could still be trouble. Suppose, for instance, that $u$ is a $J$-term, $v$ is a $K$-term, and $P$ is a binary predicate. If we tried to form $P(u, v)$ by first applying $\mathbf{S}(k / v)$ to $P(j, k)$ and then applying $\mathbf{S}(j / u)$ to the result, we could encounter an unexpected collision among the variables at the end of the first step. The point is that the term $v$ could depend on the auxiliary variable $j$ (i.e., $j$ could belong to $K$ ), and consequently $P(j, v)$ (i.e., $\mathbf{S}(k / v) P(j, k))$ could involve $j$ in a way in which $P(j, k)$ did not involve it. Here, as above, the difficulty may already arise in the case in which the roles of the terms are played by variables. (Compare $\mathbf{S}(j / i) \mathbf{S}(k / j) P(j, k)$ with $P(i, j)$.)

The difficulties of the preceding paragraph can be avoided by a judicious choice of the auxiliary variables, but there is still another objection to the 
proposed definition of $P\left(t_{1}, \cdots, t_{n}\right)$. The objection is that even if the auxiliary variables are chosen carefully, their choice might still influence the result; the proposed definition is not obviously unambiguous. We proceed to show how all these obstacles can be overcome.

(10.1) Lemma. Suppose that $J_{1}, \cdots, J_{n}$ are finite subsets of $I$, and that $t_{1}$ is a $J_{1}$-term, $\cdots, t_{n}$ is a $J_{n}$-term; write $J=J_{1} \cup \cdots \cup J_{n}$. If $i_{1}, \cdots, i_{n}$, $k_{1}, \cdots, k_{n}$ are $2 n$ distinct variables in $I-J$, and if $p$ is an element of $A$ independent of $\left\{i_{1}, \cdots, i_{n}\right\}$, then

$$
\mathbf{S}\left(i_{1} / t_{1}\right) \cdots \mathbf{S}\left(i_{n} / t_{n}\right) \mathbf{S}\left(k_{1} / i_{1}\right) \cdots \mathbf{S}\left(k_{n} / i_{n}\right) p=\mathbf{S}\left(k_{1} / t_{1}\right) \cdots \mathbf{S}\left(k_{n} / t_{n}\right) p .
$$

Proof. If $J_{0}$ is a finite subset of $I$, if $t_{0}$ is a $J_{0}$-term, and if $i, j$, and $k$ are distinct elements of $I-J_{0}$, then the transformation $(k / j)$ lives on $I-J_{0}$, and therefore, by (T5), the endomorphisms $\mathbf{S}\left(i / t_{0}\right)$ and $\mathbf{S}(k / j)$ commute with each other. By an inductive application of this comment to the left side of (10.2), the desideratum reduces to

$$
\mathbf{S}\left(i_{1} / t_{1}\right) \mathbf{S}\left(k_{1} / i_{1}\right) \cdots \mathbf{S}\left(i_{n} / t_{n}\right) \mathbf{S}\left(k_{n} / i_{n}\right) p=\mathbf{S}\left(k_{1} / t_{1}\right) \cdots \mathbf{S}\left(k_{n} / t_{n}\right) p .
$$

Let $L$ be a finite support of $p$ disjoint from $\left\{i_{1}, \cdots, i_{n}\right\}$. Since $\left(k_{n} / i_{n}\right)$ is a transformation of type $\left(\left\{k_{n}\right\}, L \cup J_{n}\right)$, and since $\left(k_{n} / i_{n}\right) k_{n}=i_{n}$, it follows from (4.1) that

$$
\mathbf{S}\left(i_{n} / t_{n}\right) \mathbf{S}\left(k_{n} / i_{n}\right) p=\mathbf{S}\left(k_{n} / t_{n}\right) p .
$$

By (7.5), $J_{n} \cup L$ supports $\mathbf{S}\left(k_{n} / t_{n}\right) p$, and, consequently, $\mathbf{S}\left(k_{n} / t_{n}\right) p$ is independent of $\left\{i_{1}, \cdots, i_{n}\right\}$. Since this comment prepares the ground for the induction step, (10.3) follows from an $n$-fold repetition of the argument just given.

(10.4) Lemma. Suppose that $J_{1}, \cdots, J_{n}$ are finite subsets of $I$, that $t_{1}$ is a $J_{1}$-term, $\cdots, t_{n}$ is a $J_{n}$-term, and that $P$ is an $n$-place predicate; write $J=J_{1} \cup \cdots \cup J_{n}$. If $i_{1}, \cdots, i_{n}$ are distinct variables in $I-J$, and $j_{1}, \cdots, j_{n}$ are distinct variables in $I-J$, then

(10.5) $\mathbf{S}\left(i_{1} / t_{1}\right) \cdots \mathbf{S}\left(i_{n} / t_{n}\right) P\left(i_{1}, \cdots, i_{n}\right)=\mathbf{S}\left(j_{1} / t_{1}\right) \cdots \mathbf{S}\left(j_{n} / t_{n}\right) P\left(j_{1}, \cdots, j_{n}\right)$.

Proof. Let $k_{1}, \cdots, k_{n}$ be distinct variables in $I-\left(J \cup\left\{i_{1}, \cdots, i_{n}\right\}\right.$ u $\left.\left\{j_{1}, \cdots, j_{n}\right\}\right)$, and write $P\left(k_{1}, \cdots, k_{n}\right)=p$. Since $\left(k_{1} / i_{1}\right) \cdots\left(k_{n} / i_{n}\right)$ $=\left(k_{1}, \cdots, k_{n} / i_{1}, \cdots, i_{n}\right)$, it follows from (2.1) that

$$
P\left(i_{1}, \cdots, i_{n}\right)=\mathbf{S}\left(k_{1} / i_{1}\right) \cdots \mathbf{S}\left(k_{n} / i_{n}\right) p .
$$

By (10.6) and (10.1), the left side of (10.5) is equal to $\mathbf{S}\left(k_{1} / t_{1}\right) \cdots \mathbf{S}\left(k_{n} / t_{n}\right) p$; the same argument with $j_{1}, \cdots, j_{n}$ in place $i_{1}, \cdots, i_{n}$ shows that the right side of (10.5) is equal to the same thing.

We are now in a position to make the promised definition. If $P$ is an $n$-place predicate, and if $t_{1}$ is a $J_{1}$-term, $\cdots, t_{n}$ is a $J_{n}$-term, we write 


$$
P\left(t_{1}, \cdots, t_{n}\right)=\mathbf{S}\left(i_{1} / t_{1}\right) \cdots \mathbf{S}\left(i_{n} / t_{n}\right) P\left(i_{1}, \cdots, i_{n}\right)
$$

whenever $i_{1}, \cdots, i_{n}$ are distinct variables in $I-\left(J_{1} \cup \cdots \cup J_{n}\right)$; Lemma (10.4) guarantees that this is an unambiguous definition. We shall obtain some of the properties of $P\left(t_{1}, \cdots, t_{n}\right)$ later; for the time being we conclude the discussion by calling attention to two useful special cases.

Since every constant is a term (a $\varnothing$-term, in fact), (10.7) assigns a meaning to $P\left(c_{1}, \cdots, c_{n}\right)$ whenever $P$ is an $n$-place predicate and $c_{1}, \cdots, c_{n}$ are constants. The proviso accompanying the definition is, of course, simpler than in the general case; we have

$$
P\left(c_{1}, \cdots, c_{n}\right)=\mathbf{S}\left(i_{1} / c_{1}\right) \cdots \mathbf{S}\left(i_{n} / c_{n}\right) P\left(i_{1}, \cdots, i_{n}\right)
$$

whenever $i_{1}, \cdots, i_{n}$ are distinct variables. The general definition allows us to consider mixed cases also; if, for instance, $P$ is a 4 -place predicate, then $P(a, b, s, t)$ makes sense whenever $a$ and $b$ are constants and $s$ and $t$ are terms.

The second useful special case is the one in which some of the terms are variables. We recall (see $\$ 4$ ) that to every variable $j$ there corresponds a $j$ constant, denoted by $c_{j}$, such that

$$
\mathbf{S}\left(K / c_{j}\right)=\mathbf{S}(K / j)
$$

for every subset $K$ of $I-\{j\}$. It follows (cf. (6.3)) that to every variable $j$ there corresponds a $j$-term (i.e., a $\{j\}$-term), which we shall denote by $t_{j}$, such that

$$
\mathbf{S}\left(K / t_{j}\right)=\mathbf{S}(K / j)
$$

for every subset $K$ of $I-\{j\}$. (Here is a situation in which it is important not to identify a singleton with its single element; the term $t_{j}$ is not the same as the $j$-constant $t_{\{j\}}$ defined by (7.1). Note that, in accordance with the definition $(6.3), t_{j}=\bar{c}_{j}$ ). It is worth observing that (10.9) holds not only for subsets of $I-\{j\}$, but in fact for all finite sets (and hence for all subsets of $I$ ). Suppose, indeed, that $p$ is an element of $A$ with finite support $L, K$ is a finite subset of $I$, and $\sigma$ is a transformation of type $(K, L \cup\{j\})$. By (4.1)

$$
\mathbf{S}\left(K / t_{j}\right) p=\mathbf{S}\left(\sigma K / t_{j}\right) \mathbf{S}(\sigma) p=\mathbf{S}(\sigma K / j) \mathbf{S}(\sigma) p .
$$

If $i \epsilon L \cap K$, then $(\sigma K / j) \sigma i=j$ and $(K / j) i=j$; if $i \epsilon L-K$, then $(\sigma K / j) \sigma i=i$ and $(K / j) i=i$. This proves that the transformations $(\sigma K / j) \sigma$ and $(K / j)$ agree on $L$ (in fact they agree outside $\sigma K$ ), and this, in turn, implies that (10.9) is true for $K$.

If the transformation law (2.1) for predicates, the definition (10.7), and the equation (10.9) are combined, the result is that $P\left(t_{1}, \cdots, t_{n}\right)$ means what it should mean in case some of the $t$ 's are variables. If, in particular, $j_{1}, \cdots, j_{n}$ are variables, then

$$
P\left(t_{j_{1}}, \cdots, t_{j_{n}}\right)=P\left(j_{1}, \cdots, j_{n}\right) .
$$


The general definition allows us to consider mixed cases also; if, for instance, $P$ is a 4-place predicate, then $P\left(t_{i}, t_{j}, s, t\right)$ makes sense whenever $i$ and $j$ are variables and $s$ and $t$ are terms. There is no loss of rigor in simplifying the notation; we shall generally write $P(i, j, s, t)$ instead of $P\left(t_{i}, t_{j}, s, t\right)$. It is easy to verify that if $s$ is a $J$-term and $t$ is a $K$-term, then

$$
P(i, j, s, t)=\mathbf{S}(h / s) \mathbf{S}(k / t) P(i, j, h, k)
$$

whenever $h$ and $k$ are distinct variables in $I-(J \cup K \cup\{i, j\})$; we shall make use of such facts without any explicit reference.

11. Transforms of terms. Given a term $t$ and a transformation $\tau$, we shall define a term, to be denoted by $\tau t$, that may be thought of as the result of transforming the variables of $t$ by $\tau$. It is to be expected, of course, that $\tau t$ will depend on $\tau$ and $t$ in just about the same way as $\tau j$ depends on $\tau$ and $j$ when $j$ is a variable. We shall see, in particular, that if $t=t_{j}$ for some variable $j$ (cf. (10.9)) and if $\tau j=k$, then $\tau t=t_{k}$; in other words, $\tau t_{j}=t_{\tau j}$. We shall be able to prove also that if $P$ is an $n$-place predicate and if $t_{1}, \cdots, t_{n}$ are terms, then

$$
\mathbf{S}(\tau) P\left(t_{1}, \cdots, t_{n}\right)=P\left(\tau t_{1}, \cdots, \tau t_{n}\right) .
$$

These surely are the minimal decency conditions that should be satisfied by any reasonable definition of $\tau t$.

The clue to the desired definition is in the equation

$$
\mathbf{S}(K / \tau j) \mathbf{S}(\tau)=\mathbf{S}(\tau) \mathbf{S}\left(\tau^{-1} K / j\right),
$$

which holds whenever $j \epsilon I, K \subset I$, and $\tau$ is a transformation on $I$. (In fact $(K / \tau j)=\tau\left(\tau^{-1} K / j\right)$. Proof: $(K / \tau j) \tau$ sends $i$ onto $\tau j$ or $\tau i$ according as $i \epsilon \tau^{-1} K$ or $i \epsilon^{\prime} \tau^{-1} K$, and the same is true of $\tau\left(\tau^{-1} K / j\right)$.) If $i$ is an element of $I$ such that $\tau^{-1}\{i\}=\{i\}$, and if we replace $K$ by $\{i\}$ in (11.1), we obtain

$$
\mathbf{S}(i / \tau j) \mathbf{S}(\tau)=\mathbf{S}(\tau) \mathbf{S}(i / j) .
$$

If $p$ is an element of $\boldsymbol{A}$ such that $\tau=\delta$ on some support of $p$, then $\mathbf{S}(\tau) p=p$, and therefore (11.2) implies that

$$
\mathbf{S}(i / \tau j) p=\mathbf{S}(\tau) \mathbf{S}(i / j) p .
$$

Verbally (11.3) says (provided $\tau, i$, and $p$ satisfy the stated conditions) that if we first replace $i$ by $j$ in $p$ and then transform the variables by $\tau$, the result is the same as if we had simply replaced $i$ by $\tau j$. On intuitive grounds this seems just as reasonable if instead of $j$ we speak of an arbitrary term $t$; we may conjecture, in other words, that if $\tau t$ were properly defined, we should have

$$
\mathbf{S}(i / \tau t) p=\mathbf{S}(\tau) \mathbf{S}(i / t) p
$$


whenever $\tau^{-1}\{i\}=\{i\}$ and $\tau=\delta$ on some support of $p$. Our definition of $\tau t$ will be guided by (11.4).

One more preliminary comment is needed. If $t$ is a $J$-term, what kind of a term should $\tau t$ be? The answer is clear: if $t$ depends on the variables in $J$, and on no others, and if those variables are transformed by $\tau$, the result should depend on the variables in $\tau J$, and on no others. If $t$ is a $J$-term, it is to be expected that $\tau t$ will be a $\tau J$-term.

(11.5) LеммA. If $t$ is a $J$-term, if $\tau$ is a transformation that lives on a finite subset $K$ of $I$, and if $i$ is an element of $I-(J \cup K)$, then there exists a unique $\tau J$-term s such that

$$
\mathbf{S}(i / s) p=\mathbf{S}(\tau) \mathbf{S}(i / t) p
$$

whenever $p$ is independent of $K$.

Proof. The construction of $s$ is based on (9.2); the set $\tau J$ will play the role of what was there denoted by $J$. (Observe that since $i \in I-K$, it follows that $\tau^{-1}\{i\}=\{i\}$; since also $i \in I-J$, it follows that $i \in I-\tau J$.) If $p$ is independent of $K$, we write

$$
g p=\mathbf{S}(\tau) \mathbf{S}(i / t) p .
$$

It is clear that $g$ is a Boolean homomorphism from the range of $\exists(K)$ into $\boldsymbol{A}$; we proceed to verify that $g$ satisfies the conditions (1)-(4) of (9.2) (with $\tau J$ in place of $J$ ).

We have

$$
\begin{aligned}
\exists(i) g \exists(K) & =\exists(i) \mathbf{S}(\tau) \mathbf{S}(i / t) \exists(K)=\mathbf{S}(\tau) \exists(i) \mathbf{S}(i / t) \exists(K) \\
& =\mathbf{S}(\tau) \mathbf{S}(i / t) \exists(K)[\text { by (T4)] }=g \exists(K),
\end{aligned}
$$

and

$$
\begin{aligned}
g \exists(K) \exists(i) & =\mathbf{S}(\tau) \mathbf{S}(i / t) \exists(K) \exists(i)=\mathbf{S}(\tau) \mathbf{S}(i / t) \exists(i) \exists(K) \\
& =\mathbf{S}(\tau) \exists(i) \exists(K)[\text { by (T3) and (T1)]= }=\exists(i) \mathbf{S}(\tau) \exists(K) \\
& =\exists(i) \exists(K) \text { [since } \tau \text { lives on } K]=\exists(K) \exists(i)
\end{aligned}
$$

this settles (9.2)(1) and (9.2)(2).

Suppose next that $j$ is an element of $I-\tau J$ such that $j \neq i$. We split the discussion into two cases, according as $j \epsilon K$ or $j \epsilon^{\prime} K$. If $j \epsilon K$, and if $p$ is an arbitrary element of $\boldsymbol{A}$, then $I-K$ supports $\exists(K) p$ and therefore $J \mathbf{u}(I-K)$ supports $\mathbf{S}(i / t) \exists(K) p(7.5)$. It follows that $\tau(J \mathbf{u}(I-K))$ supports $g \exists(K) p$. Since $\tau(J \cup(I-K))=\tau J \cup(I-K)$, and since $j$ does not belong to this set, $g \exists(K) p$ is independent of $j$; consequently

$$
g \exists(K) \exists(j) p=g \exists(K) p \text { [since } j \epsilon K]=\exists(j) g \exists(K) p .
$$

If $j \epsilon^{\prime} K$, then $\tau j=j$ and therefore (since $\left.j \epsilon^{\prime} \tau J\right) j \epsilon^{\prime} J$. It follows that 


$$
\begin{aligned}
g \exists(K) \exists(j) & =\mathbf{S}(\tau) \mathbf{S}(i / t) \exists(K) \exists(j)=\mathbf{S}(\tau) \mathbf{S}(i / t) \exists(j) \exists(K) \\
& =\mathbf{S}(\tau) \exists(j) \mathbf{S}(i / t) \exists(K)[\text { by (T3)] } \\
& =\exists(j) \mathbf{S}(\tau) \mathbf{S}(i / t) \exists(K)\left[\text { since } j \epsilon^{\prime} K\right]=\exists(j) g \exists(K) ;
\end{aligned}
$$

this concludes the proof of (9.2)(3).

Suppose finally that $\sigma$ is a finite transformation that lives on $I-(\{i\}$ $\cup \tau J \cup K$ ). It follows from (8.7) (with $\sigma$ and $\{i\}$ in the roles of what are there denoted by $\tau$ and $K)$ that $\mathbf{S}(i / t)$ commutes with $\mathbf{S}(\sigma)$. Since $\tau$ lives on $K$, and since this implies that $\tau$ commutes with $\sigma$, it follows that

$$
\begin{aligned}
g \exists(K) \mathbf{S}(\sigma) & =\mathbf{S}(\tau) \mathbf{S}(i / t) \exists(K) \mathbf{S}(\sigma) \\
& =\mathbf{S}(\tau) \mathbf{S}(i / t) \mathbf{S}(\sigma) \exists(K) \text { [since } \sigma \text { lives on } I-K] \\
& =\mathbf{S}(\sigma) \mathbf{S}(\tau) \mathbf{S}(i / t) \exists(K)=\mathbf{S}(\sigma) g \exists(K) .
\end{aligned}
$$

The existence of the $\tau J$-term $s$ satisfying (11.6) is now an immediate consequence of (9.2); uniqueness is guaranteed by (9.8).

We prove next that the term $s$ of (11.5) does not depend on the choice of $i$ and $K$.

(11.7) Lemma. Suppose that $t$ is a J-term, that $\tau$ is a transformation that lives on both $H$ and $K$ (where $H$ and $K$ are finite subsets of $I$ ), and that $i$ and $j$ are elements of $I-(J \cup H)$ and $I-(J \cup K)$ respectively. If $u$ and $v$ are $\tau J$-terms such that

$$
\mathbf{S}(i / u) p=\mathbf{S}(\tau) \mathbf{S}(i / t) p
$$

whenever $p$ is independent of $H$ and

$$
\mathbf{S}(j / v) p=\mathbf{S}(\tau) \mathbf{S}(j / t) p
$$

whenever $p$ is independent of $K$, then $u=v$.

Proof. Since $\tau$ lives on $H \cap K$ and since $i$ and $j$ are in $I-(H \cap K)$, it follows that $\tau$ commutes with the transformation $(i, j)$. We observe that $(i, j)$ lives on both $I-J$ and $I-\tau J$, and that if $p$ is independent of $H \cup K$ (so that, in particular, $p$ is independent of both $H$ and $K)$, then $\mathbf{S}(i, j) p=p$. It follows that

$$
\begin{aligned}
\mathbf{S}(i / u) p & =\mathbf{S}(\tau) \mathbf{S}(i / t) p=\mathbf{S}(\tau) \mathbf{S}(i / t) \mathbf{S}(i, j) p \\
& =\mathbf{S}(\tau) \mathbf{S}(i, j) \mathbf{S}(j / t) p[\text { by (T5)] }=\mathbf{S}(i, j) \mathbf{S}(\tau) \mathbf{S}(j / t) p \\
& =\mathbf{S}(i . i) \mathbf{S}(j / v) p=\mathbf{S}(i / v) \mathbf{S}(i, j) p[\text { by (T5)] }=\mathbf{S}(i / v) p
\end{aligned}
$$

the desired equality is now a consequence of (9.8).

We are now prepared to define $\tau t$ whenever $t$ is a $J$-term and $\tau$ is a finite transformation. Let $K$ be a finite set such that $\tau$ lives on $K$ and let $i$ be an element of $I-(J \cup K)$. We define $\tau t$ to be the unique $\tau J$-term obtained by applying (11.5) to this situation, so that 


$$
\mathbf{S}(i / \tau t) p=\mathbf{S}(\tau) \mathbf{S}(i / t) p
$$

whenever $p$ is independent of $K$. Lemma (11.7) guarantees that the definition is unambiguous (i.e., that as long as $K$ and $i$ satisfy the stated conditions, it does not matter how they are chosen). We proceed to prove that this definition has all the reasonable properties we promised for it.

(11.9) Lemma. If $t$ is a $J$-term and if $\sigma$ and $\tau$ are finite transformations such that $\sigma=\tau$ on $J$, then $\sigma t=\tau t$.

Proof. Select a finite set $K$ such that both $\sigma$ and $\tau$ live on $K$ and let $i$ be an element of $I-(J \cup K)$. If $p$ is independent of $K$, then

$$
\mathbf{S}(i / \sigma t) p=\mathbf{S}(\sigma) \mathbf{S}(i / t) p \text { and } \mathbf{S}(i / \tau t) p=\mathbf{S}(\tau) \mathbf{S}(i / t) p .
$$

Since $J \mathbf{u}(I-K)$ supports $\mathbf{S}(i / t) p$ and since $\sigma=\tau$ on $J \mathbf{u}(I-K)$, it follows that $\mathbf{S}(i / \sigma t) p=\mathbf{S}(i / \tau t) p$; the desired equality is now a consequence of (9.8).

Using (11.9), we can extend the definition of $\tau t$ to the case of a not necessarily finite $\tau$, as follows. If $t$ is a $J$-term and if $\tau$ is a transformation, let $\sigma$ be an arbitrary finite transformation such that $\tau=\sigma$ on $J$, and write $\tau t=\sigma t$. If $\sigma_{1}$ and $\sigma_{2}$ are finite transformations such that $\tau=\sigma_{1}$ in $J$ and $\tau=\sigma_{2}$ in $J$, then $\sigma_{1} t=\sigma_{2} t$ (by (11.9)) and therefore the definition of $\tau t$ is unambiguous. If, in particular, $\tau$ happens to be finite, the new definition of $\tau t$ is compatible with the old one. Observe that since $\sigma J=\tau J$, the term $\tau t$ is always a $\tau J$-term.

(11.10) THEOREM. If $t$ is a term, then

$$
\delta t=t
$$

if $\sigma$ and $\tau$ are transformations, then

$$
(\sigma \tau) t=\sigma(\tau t) .
$$

If $t$ is a $J$-term, then

$$
\sigma t=\tau t \text { whenever } \sigma=\tau \text { on } J .
$$

Proof. Suppose that $t$ is a $J$-term. Let $i$ be an element of $I-J$. Since $\delta$ lives on $\varnothing$, it follows that

$$
\mathbf{S}(i / \delta t) p=\mathbf{S}(\delta) \mathbf{S}(i / t) p=\mathbf{S}(i / t) p
$$

for all $p$, and hence $(9.8)$ that $\delta t=t$.

To prove (11.12), write $\rho=\sigma \tau$ and define the auxiliary transformations $\rho_{0}$, $\sigma_{0}$, and $\tau_{0}$, as follows:

$$
\begin{array}{ll}
\rho_{0}=\rho \text { on } J, & \rho_{0}=\delta \text { on } I-J, \\
\sigma_{0}=\sigma \text { on } \tau J, & \sigma_{0}=\delta \text { on } I-\tau J, \\
\tau_{0}=\tau \text { on } J, & \tau_{0}=\delta \text { on } I-J .
\end{array}
$$

The transformations $\rho_{0}, \sigma_{0}$, and $\tau_{0}$ are finite; it follows from the definition of 
the action of a not necessarily finite transformation that $\rho t=\rho_{0} t, \tau t=\tau_{0} t$, and $\sigma(\tau t)=\sigma_{0}(\tau t)$.

Let $K$ be a finite set such that $\rho_{0}, \sigma_{0}$, and $\tau_{0}$ live on $K$, and let $i$ be an element of $I-(J \cup K)$. Since $\sigma_{0} \tau_{0}$ lives on $K$, it follows that if $p$ is independent of $K$, then

$$
\begin{aligned}
\mathbf{S}\left(i /\left(\sigma_{0} \tau_{0}\right) t\right) p & =\mathbf{S}\left(\sigma_{0} \tau_{0}\right) \mathbf{S}(i / t) p=\mathbf{S}\left(\sigma_{0}\right) \mathbf{S}\left(\tau_{0}\right) \mathbf{S}(i / t) p \\
& =\mathbf{S}\left(\sigma_{0}\right) \mathbf{S}\left(i / \tau_{0} t\right) p=\mathbf{S}\left(i / \sigma_{0}\left(\tau_{0} t\right)\right) p .
\end{aligned}
$$

The uniqueness theorem (9.8) implies now that $\left(\sigma_{0} \tau_{0}\right) t=\sigma_{0}\left(\tau_{0} t\right)$. The transformations $\rho_{0}$ and $\sigma_{0} \tau_{0}$ are not necessarily equal, but it is easy to verify that they agree on $J$; it follows that

$$
(\sigma \tau) t=\rho t=\rho_{0} t=\left(\sigma_{0} \tau_{0}\right) t=\sigma_{0}\left(\tau_{0} t\right)=\sigma_{0}(\tau t)=\sigma(\tau t) .
$$

The last assertion of the theorem (i.e., (11.13)) is immediate from the definitions.

(11.14) THEOREM. If $\tau$ is a transformation and if $j$ is a variable, then $\tau t_{j}=t_{\tau j}$

Proof. It is sufficient to consider finite transformations only. Let $K$ be a finite set such that $\tau$ lives on $K$. If $i \in I-(J \cup K)$ and if $k \in I-K$, then either $k=i$, in which case

$$
(i / \tau j) k=(i / \tau j) i=\tau j=\tau(i / j) i=\tau(i / j) k,
$$

or else $k \neq i$, in which case

$$
(i / \tau j) k=k=\tau k=\tau(i / j) k .
$$

Hence, in either case, $(i / \tau j)=\tau(i / j)$ outside $K$; it follows that if $p$ is independent of $K$, then $\mathbf{S}(i / \tau j) p=\mathbf{S}(\tau) \mathbf{S}(i / j) p$.

(11.15) THEOREM. If $t$ is a term, if $\tau$ is a transformation, and if $i$ is an element of $I$ such that $\tau^{-1}\{i\}=\{i\}$, then

$$
\mathbf{S}(i / \tau t) \mathbf{S}(\tau)=\mathbf{S}(\tau) \mathbf{S}(i / t) .
$$

Proof. Suppose that $t$ is a $J$-term. Let $p$ be an element of $A$ with finite support $L$; observe that $L \cup J$ supports $\mathbf{S}(i / t) p$. If $\tau_{0}=\tau$ on $L \cup J$ and $\tau_{0}=\delta$ outside $L \cup J$, then $\tau_{0}$ is a finite transformation such that $\tau_{0}^{-r}\{i\}=\{i\}, \tau_{0} t=\tau t$, $\mathbf{S}\left(\tau_{0}\right) p=\mathbf{S}(\tau) p$, and $\mathbf{S}\left(\tau_{0}\right) \mathbf{S}(i / t) p=\mathbf{S}(\tau) \mathbf{S}(i / t) p$. It follows that there is no loss of generality in assuming (as we do from now on) that the given transformation $\tau$ is finite. Let $K$ be a finite set not containing $i$ such that $\tau$ lives on $K$.

Assume now that $i \in I-J$. Let $\sigma$ be a transformation of type $(K,\{i\} \cup J$ $u L$ ). If $\rho$ is the transformation such that $\rho=\delta$ outside $\sigma K$ and $\rho k=\tau \tilde{\sigma} k$ when $k \in \sigma K$, then $\tau \tilde{\sigma}=\rho \tau$. By (8.7) (first with $\tau t, \tau J, \rho$, and $\{i\}$, and then with $t, J, \tilde{\sigma}$, and $\{i\}$ in the roles of $t, J, \tau$, and $K$, respectively) it follows that $\mathbf{S}(\rho)$ 
commutes with $\mathbf{S}(i / \tau t)$ and $\mathbf{S}(\tilde{\boldsymbol{\sigma}})$ commutes with $\mathbf{S}(i / t)$. Consequently

$$
\begin{aligned}
\mathbf{S}(i / \tau t) \mathbf{S}(\tau) p & =\mathbf{S}(i / \tau t) \mathbf{S}(\tau) \mathbf{S}(\tilde{\sigma}) p \\
& =\mathbf{S}(i / \tau t) \mathbf{S}(\tau) \mathbf{S}(\tilde{\sigma}) \mathbf{S}(\sigma) p[\text { by }(3.3)] \\
& =\mathbf{S}(i / \tau t) \mathbf{S}(\rho) \mathbf{S}(\tau) \mathbf{S}(\sigma) p=\mathbf{S}(\rho) \mathbf{S}(i / \tau t) \mathbf{S}(\tau) \mathbf{S}(\sigma) p \\
& =\mathbf{S}(\rho) \mathbf{S}(\tau) \mathbf{S}(i / t) \mathbf{S}(\sigma) p[\text { since } \mathbf{S}(\sigma) p \text { is independent of } K] \\
& =\mathbf{S}(\tau) \mathbf{S}(\tilde{\sigma}) \mathbf{S}(i / t) \mathbf{S}(\sigma) p=\mathbf{S}(\tau) \mathbf{S}(i / t) \mathbf{S}(\tilde{\sigma}) \mathbf{S}(\sigma) p \\
& =\mathbf{S}(\tau) \mathbf{S}(i / t) p
\end{aligned}
$$

this completes the proof in case $i \epsilon I-J$.

If $i \epsilon J$, let $j$ be an element of $I-(L \cup J \cup K)$. The transformation $(i / j)$ is of type $(\{i\}, L \cup J)$ and also of type $(\{i\}, \tau L \cup \tau J)$; it follows from (4.1) that

$$
\mathbf{S}(i / t) p=\mathbf{S}(j / t) \mathbf{S}(i / j) p
$$

and

$$
\begin{aligned}
\mathbf{S}(i / \tau t) \mathbf{S}(\tau) p & =\mathbf{S}(i / \tau t) \mathbf{S}(i / j) \mathbf{S}(\tau) p \\
& =\mathbf{S}(j / \tau t) \mathbf{S}(\tau) \mathbf{S}(i / j) p[\text { since }\{i, j\} \subset I-K] \\
& =\mathbf{S}(\tau) \mathbf{S}(j / t) \mathbf{S}(i / j) p[\text { since } j \epsilon I-J] \\
& =\mathbf{S}(\tau) \mathbf{S}(i / t) p[\text { by }(11.16)] .
\end{aligned}
$$

This completes the proof of Theorem (11.15).

We show finally that the process of replacing the arguments of a predicate by terms is decently related to the action of transformations on terms.

(11.17) Theorem. If $P$ is an $n$-place predicate, if $t_{1}, \cdots, t_{n}$ are terms, and if $\tau$ is a transformation, then

$$
\mathbf{S}(\tau) P\left(t_{1}, \cdots, t_{n}\right)=P\left(\tau t_{1}, \cdots, \tau t_{n}\right) .
$$

Proof. Suppose that $t_{1}$ is a $J_{1}$-term, $\cdots, t_{n}$ is a $J_{n}$-term, and write $J=J_{1} \cup \cdots \cup J_{n}$. Let $\tau_{0}$ be the transformation that agrees with $\tau$ on $J$ and that is equal to $\delta$ outside $J$. Since the desired conclusion is the same for $\tau$ as for $\tau_{0}$, we may henceforth assume that the transformation $\tau$ is finite to begin with.

If $i_{1}, \cdots, i_{n}$ are distinct elements of $I-(J \cup \tau J)$, such that $\tau^{-1}\left\{i_{1}\right\}$ $=\left\{i_{1}\right\}, \cdots, \tau^{-1}\left\{i_{n}\right\}=\left\{i_{n}\right\}$, then

$$
\begin{aligned}
\mathbf{S}(\tau) P\left(t_{1}, \cdots, t_{n}\right) & =\mathbf{S}(\tau) \mathbf{S}\left(i_{1} / t_{1}\right) \cdots \mathbf{S}\left(i_{n} / t_{n}\right) P\left(i_{1}, \cdots, i_{n}\right)[\text { by }(10.7)] \\
& =\mathbf{S}\left(i_{1} / \tau t_{1}\right) \cdots \mathbf{S}\left(i_{n} / \tau t_{n}\right) \mathbf{S}(\tau) P\left(i_{1}, \cdots, i_{n}\right)[\text { by }(11.15)] \\
& =\mathbf{S}\left(i_{1} / \tau t_{1}\right) \cdots \mathbf{S}\left(i_{n} / \tau t_{n}\right) P\left(i_{1}, \cdots, i_{n}\right)[\text { by }(2.1)] \\
& =P\left(\tau t_{1}, \cdots, \tau t_{n}\right) \quad[\text { by }(10.7)] .
\end{aligned}
$$

This concludes our discussion of the transforms of terms. 
12. Operations. An operation (as we shall define it) is something that converts variables into $J$-terms, in about the same way as a predicate converts variables into $J$-propositions. Equality, for instance, is a binary predicate; suitably combined with the variables $x$ and $y$ it becomes the $\{x, y\}$-proposition $x=y$. Similarly, addition is a binary operation; suitably combined with the variables $x$ and $y$ it becomes the $\{x, y\}$-term $x+y$. Using the facts about terms and their transforms derived in the preceding sections, we can obtain the basic theory of operations quite easily; the techniques are minor modifications of the ones used at the corresponding parts of the theory of predicates.

We begin with an auxiliary result (a corollary of (9.2)) that enables us to tell when a term is a $J$-term.

(12.1) Lemma. If $J$ and $K$ are finite subsets of $I$, if $t$ is a $(J \cup K)$-term, and if $i$ is an element of $I-(J \cup K)$ such that

$$
\mathbf{S}(i / t) \exists(K) \exists(j)=\exists(j) \mathbf{S}(i / t) \exists(K) \text { whenever } j \neq i \text { and } j \in I-J,
$$

then $t$ is a $J$-term.

Proof. Write $g p=\mathbf{S}(i / t) p$ whenever $p$ is independent of $K$. Clearly $g$ is a Boolean homomorphism from the range of $\exists(K)$ into $A$; we shall prove that $g$ satisfies the conditions (1)-(4) of (9.2). We have

$$
\exists(i) g \exists(K)=\exists(i) \mathbf{S}(i / t) \exists(K)=\mathbf{S}(i / t) \exists(K)[\text { by (T4)] }=g \exists(K),
$$

and

$$
\begin{aligned}
g \exists(K) \exists(i) & =\mathbf{S}(i / t) \exists(K) \exists(i)=\mathbf{S}(i / t) \exists(i) \exists(K) \\
& =\exists(i)(K)[\text { by (T3) and (T1)] }=\exists(K) \exists(i)
\end{aligned}
$$

this, together with (12.2), settles (9.2)(1), (9.2)(2), and (9.2)(3). If $\tau$ is a finite transformation that lives on $I-(\{i\} \cup J \cup K)$, then

$$
\begin{aligned}
g \exists(K) \mathbf{S}(\tau) & =\mathbf{S}(i / t) \exists(K) \mathbf{S}(\tau)=\mathbf{S}(i / t) \mathbf{S}(\tau) \exists(K)\left[\text { since } \tau^{-1} K=K\right] \\
& =\mathbf{S}(\tau) \mathbf{S}(i / t) \exists(K)\left[\text { by (T5), since } \tau^{-1}\{i\}=\{i\}\right]=\mathbf{S}(\tau) g \exists(K)
\end{aligned}
$$

this settles (9.2)(4). It follows from (9.2) that there exists a $J$-term $s$ such that $\mathbf{S}(i / s) p=\mathbf{S}(i / t) p$ whenever $p$ is independent of $K$. Since $s=t$ (by (9.8)), it follows that $t$ is a $J$-term, as asserted.

An $n$-place (or $n$-ary) operation $(n=1,2,3, \cdots)$ of an $I$-algebra $A$ is a function $T$ on $I^{n}$ whose values are terms of $A$, such that if $\left(i_{1}, \cdots, i_{n}\right) \in I^{n}$ and if $\tau$ is a transformation on $I$, then

$$
\tau T\left(i_{1}, \cdots, i_{n}\right)=T\left(\tau i_{1}, \cdots, \tau i_{n}\right) .
$$

We shall sometimes say that an $n$-place operation is an operation of degree $n$.

We prove now that the analogues of (2.2), (2.3), and (2.4) are valid for operations. 
(12.4) TheOREM. If $T$ is an $n$-place operation and if $\left(i_{1}, \cdots, i_{n}\right) \in I^{n}$, then $T\left(i_{1}, \cdots, i_{n}\right)$ is an $\left\{i_{1}, \cdots, i_{n}\right\}$-term.

Proof. Write $t=T\left(i_{1}, \cdots, i_{n}\right)$. We know that $t$ is a term; let us say that $t$ is a $\bar{J}$-term. If $J=\left\{i_{1}, \cdots, i_{n}\right\}$ and $K=\bar{J}-J$, then $\bar{J} \subset J \cup K$ and therefore $t$ is a $(J \cup K)$-term. We shall prove that $t$ is a $J$-term by applying (12.1). Suppose, accordingly, that $i \in I-(J \cup K)$ and that $j$ is an element of $I-J$ distinct from $i$; it is sufficient to prove (cf. (12.2)) that

$$
\mathbf{S}(i / t) \exists(j)=\exists(j) \mathbf{S}(i / t) .
$$

For this purpose, let $k$ be an element of $I-(J \cup K)$ distinct from both $i$ and $j$. Since $(j, k)^{-1}\{i\}=\{i\}$, and since (by (12.3)) $(j, k) t=t$, it follows from (11.15) that

$$
\mathbf{S}(i / t) \mathbf{S}(j, k)=\mathbf{S}(j, k) \mathbf{S}(i / t)
$$

Consequently

$$
\begin{aligned}
\mathbf{S}(i / t) \exists(j) & =\mathbf{S}(i / t) \mathbf{S}(j, k) \exists(k) \mathbf{S}(j, k) \\
& =\mathbf{S}(j, k) \mathbf{S}(i / t) \exists(k) \mathbf{S}(j, k)[\text { by }(12.5)] \\
& =\mathbf{S}(j, k) \exists(k) \mathbf{S}(i / t) \mathbf{S}(j, k)[\text { by }(\mathrm{T} 3)] \\
& =\mathbf{S}(j, k) \exists(k) \mathbf{S}(j, k) \mathbf{S}(i / t)[\text { by }(12.5)]=\exists(j) \mathbf{S}(i / t),
\end{aligned}
$$

and the proof of the theorem is complete.

(12.6) Lemma. If $j_{1}, \cdots, j_{n}$ are distinct elements of $I$ and if $t$ is a $\left\{j_{1}, \cdots, j_{n}\right\}$-term, then there exists a unique $n$-place operation $T$ such that $T\left(j_{1}, \cdots, j_{n}\right)=t$.

Proof. The operation $T$ is defined by

$$
T\left(i_{1}, \cdots, i_{n}\right)=\left(j_{1}, \cdots, j_{n} / i_{1}, \cdots, i_{n}\right) t .
$$

To prove that the function $T$ is indeed an operation, let $\tau$ be an arbitrary transformation on $I$ and note that if $j \in\left\{j_{1}, \cdots, j_{n}\right\}$, then

$$
\tau \cdot\left(j_{1}, \cdots, j_{n} / i_{1}, \cdots, i_{n}\right) j=\left(j_{1}, \cdots, j_{n} / \tau i_{1}, \cdots, \tau i_{n}\right) j .
$$

Since, by assumption, $t$ is a $\left\{j_{1}, \cdots, j_{n}\right\}$-term, it follows (cf. (11.13)) that

$$
\begin{aligned}
\tau T\left(i_{1}, \cdots, i_{n}\right) & =\tau \cdot\left(j_{1}, \cdots, j_{n} / i_{1}, \cdots, i_{n}\right) t \\
& =\left(j_{1}, \cdots, j_{n} / \tau i_{1}, \cdots, \tau i_{n}\right) t=T\left(\tau i_{1}, \cdots, \tau i_{n}\right) .
\end{aligned}
$$

The uniqueness of $T$ is an immediate consequence of (12.3), with

$$
\left(j_{1}, \cdots, j_{n} / i_{1}, \cdots, i_{n}\right)
$$

in the role of $\tau$.

(12.7) Corollary. Every term of $\boldsymbol{A}$ is in the range of some operation of $\boldsymbol{A}$. 
It is reasonable to ask whether the concept of an operation can be generalized the same way as the concept of a $J$-predicate generalizes the concept of a predicate. The answer is yes, but since neither the techniques nor the results of the generalized theory offer any points of novelty, we do not enter into the details.

We conclude this preliminary discussion of operations by observing that there is a natural way of combining predicates and operations to form predicates of higher degree. If $P$ is a predicate of degree $n$, and if $T_{1}, \cdots, T_{n}$ are operations of degrees $m_{1}, \cdots, m_{n}$ respectively, we write $m=m_{1}+\cdots+m_{n}$ and we define a predicate $Q$ of degree $m$ by writing $Q=P\left(T_{1}, \cdots, T_{n}\right)$. To be more precise, suppose that $P$ is a binary predicate and that $S$ and $T$ are operations of degrees $m$ and $n$ respectively; in that case the predicate $P(S, T)$ $(=Q)$ is the $(n+m)$-place predicate defined by

$$
Q\left(i_{1}, \cdots, i_{m}, j_{1}, \cdots, j_{n}\right)=P\left(S\left(i_{1}, \cdots, i_{m}\right), T\left(j_{1}, \cdots, j_{n}\right)\right) .
$$

(The only essential difference between this special case and the most general case is that for the latter the number of indices that it is necessary to use makes the theory appear much more complicated than it really is.) If $\tau$ is a transformation on $I$, then

$$
\begin{aligned}
\mathbf{S}(\tau) Q\left(i_{1}, \cdots,\right. & \left.i_{m}, j_{1}, \cdots, j_{n}\right) \\
& =P\left(\tau S\left(i_{1}, \cdots, i_{m}\right), \tau T\left(j_{1}, \cdots, j_{n}\right)\right)[\text { by }(11.17)] \\
& =P\left(S\left(\tau i_{1}, \cdots, \tau i_{m}\right), T\left(\tau j_{1}, \cdots, \tau j_{n}\right)\right)[\text { by }(12.3)] \\
& =Q\left(\tau i_{1}, \cdots, \tau i_{m}, \tau j_{1}, \cdots, \tau j_{n}\right) ;
\end{aligned}
$$

this proves that the function $Q$ is indeed a predicate.

13. Terms in terms. Since transformations act on elements $p$ of $A$ and, as we have seen, on terms $t$ of $A$, it makes sense to speak of replacing some variables by others in $p$ or in $t$. It also makes sense to speak of replacing variables by terms in $p$; our next purpose is to discuss the analogous process of replacing variables by terms in a term $t$. Given two terms $s$ and $t$ and a subset $K$ of $I$, we shall define a term, to be denoted by $(K / s) t$, that depends on $s$ in just about the same way as the image of $t$ under the transformation $(K / j)$, i.e., $(K / j) t$, depends on the variable $j$. The definition of $(K / s) t$ is similar in spirit, and even in some details, to the definition (cf. \$11) of the way a transformation acts on a term.

(13.1) Lemma. If $s$ is an $H$-term, $t$ is a $J$-term, $K$ is a finite subset of $I$, and $i$ is an element of $I-(H \cup J \cup K)$, then there exists a unique $(H \cup(J-K))$ term $u$ such that

$$
\mathbf{S}(i / u) p=\mathbf{S}(K / s) \mathbf{S}(i / t) p
$$

whenever $p$ is independent of $K$. 
Proof. The plan of attack is similar to the one used in (11.5). If $p$ is indepent of $K$, we write

$$
g p=\mathbf{S}(K / s) \mathbf{S}(i / t) p .
$$

We construct $u$ by applying (9.2); the set $\bar{J}=H \cup(J-K)$ will play the role of what was there denoted by $J$. Since $\bar{J} \cup K=H \cup J \cup K$, it follows from our assumption concerning $i$ that $i \epsilon I-(\bar{J} \cup K)$. It is clear that $g$ is a Boolean homomorphism from the range of $\exists(K)$ into $A$; we proceed to verify that $g$ satisfies the conditions (1)-(4) of (9.2) (with $\bar{J}$ in place of $J$ ).

We have

$$
\begin{aligned}
& \exists(i) g \exists(K)=\exists(i) \mathbf{S}(K / s) \mathbf{S}(i / t) \exists(K)=\mathbf{S}(K / s) \exists(i) \mathbf{S}(i / t) \exists(K) \text { [by }(\overline{\mathbf{T}} 4) \text { ] } \\
& =\mathbf{S}(K / s) \mathbf{S}(i / t) \exists(K)[\text { by (T4)] }=g \exists(K)
\end{aligned}
$$

and

$$
\begin{aligned}
g \exists(K) \exists(i) & =\mathbf{S}(K / s) \mathbf{S}(i / t) \exists(K) \exists(i)=\mathbf{S}(K / s) \mathbf{S}(i / t) \exists(i) \exists(K) \\
& =\mathbf{S}(K / s) \exists(i) \exists(K)[\text { by (T3) }]=\mathbf{S}(K / s) \exists(K) \exists(i)=\exists(K) \exists(i) .
\end{aligned}
$$

The justification of the last equation is that the image under $\exists(K)$ of every element of $\boldsymbol{A}$ has $I-K$ for a support, and consequently, by (7.6), $\mathbf{S}(K / s) \exists(K)$ $=\mathbf{S}(\varnothing / s) \exists(K)$. This settles $(9.2)(1)$ and $(9.2)(2)$.

Suppose next that $j$ is an element of $I-\bar{J}$ (i.e., that $\left.j \epsilon^{\prime} H \mathbf{u}(J-K)\right)$ and that $j \neq i$. We split the discussion into two cases, according as $j \epsilon K$ or $j \epsilon^{\prime} K$. If $j \epsilon K$, and if $p$ is an arbitrary element of $A$, then $I-K$ supports $\exists(K) p$, and therefore $J \mathbf{u}(I-K)$ supports $\mathbf{S}(i / t) \exists(K) p$ (7.5). It follows (again by (7.5)) that $H \cup(I-K)$ supports $g \exists(K) p$. Since $j$ does not belong to this set, $g \exists(K) p$ is independent of $j$; consequently

$$
g \exists(K) \exists(j) p=g \exists(K) p[\text { since } j \in K]=\exists(j) g \exists(K) p .
$$

If $j \epsilon^{\prime} K$, then $j \epsilon^{\prime} H \cup J$; it follows that

$$
\begin{aligned}
g \exists(K) & \exists(j)=\mathbf{S}(K / s) \mathbf{S}(i / t) \exists(K) \exists(j)=\mathbf{S}(K / s) \mathbf{S}(i / t) \exists(j) \exists(K) \\
& =\mathbf{S}(K / s) \exists(j) \mathbf{S}(i / t) \exists(K)[\text { by (T3)]= } \exists(j) \mathbf{S}(K / s) \mathbf{S}(i / t) \exists(K)[\text { by (T) } 3) \\
& =\exists(j) g \exists(K) ;
\end{aligned}
$$

this concludes the proof of $(9.2)(3)$.

Suppose finally that $\tau$ is a finite transformation that lives on $I-(\{i\}$ $\cup \bar{J} \cup K)$. It follows that

$$
\begin{aligned}
g \exists(K) \mathbf{S}(\tau) & =\mathbf{S}(K / s) \mathbf{S}(i / t) \exists(K) \mathbf{S}(\tau) \\
& =\mathbf{S}(K / s) \mathbf{S}(i / t) \mathbf{S}(\tau) \exists(K) \quad[\text { since } \tau \text { lives on } I-K] \\
& =\mathbf{S}(K / s) \mathbf{S}(\tau) \mathbf{S}(i / t) \exists(K)[\text { by (T5)] } \\
& =\mathbf{S}(\tau) \mathbf{S}(K / s) \mathbf{S}(i / t) \exists(K)[\text { by (T) } 5)]=\mathbf{S}(\tau) g \exists(K) .
\end{aligned}
$$


The existence of a $\bar{J}$-term $u$ satisfying (13.2) is now an immediate consequence of (9.2); uniqueness is guaranteed by (9.8).

We prove next that the term $u$ of (13.1) does not depend on the choice $i$.

(13.3) Lemma. Suppose that $s$ is an H-term, $t$ is a J-term, $K$ is a finite subset of $I$, and $i$ and $j$ are elements of $I-(H \cup J \cup K)$. If $u$ and $v$ are $(H \cup(J$ $-K))$-terms such that

$$
\mathbf{S}(i / u) p=\mathbf{S}(K / s) \mathbf{S}(i / t) p \quad \text { and } \quad \mathbf{S}(j / v) p=\mathbf{S}(K / s) \mathbf{S}(j / t) p
$$

whenever $p$ is independent of $K$, then $u=v$.

Proof. Since the transformation $(i, j)$ lives on $I-K$, it follows that if $p$ is independent of $K$, then $\mathbf{S}(i, j) p=p$. This implies that

$$
\begin{aligned}
\mathbf{S}(i / u) p & =\mathbf{S}(K / s) \mathbf{S}(i / t) p=\mathbf{S}(K / s) \mathbf{S}(i / t) \mathbf{S}(i, j) p \\
& =\mathbf{S}(K / s) \mathbf{S}(i, j) \mathbf{S}(j / t) p[\text { by }(\mathrm{T} 5)]=\mathbf{S}(i, j) \mathbf{S}(K / s) \mathbf{S}(j / t) p[\text { by }(\overline{\mathrm{T}} 5)] \\
& =\mathbf{S}(i, j) \mathbf{S}(j / v) p=\mathbf{S}(j / v) \mathbf{S}(i, j) p[\text { by }(\mathrm{T} 5)]=\mathbf{S}(j / v) p ;
\end{aligned}
$$

the desired equality is now a consequence of (9.8).

We are now prepared to define $(K / s) t$ whenever $s$ is an $H$-term, $t$ is a $J$-term, and $K$ is a finite set. Let $i$ be an element of $I-(H \cup J \cup K)$, and define $(K / s) t$ to be the unique $(H \cup(J-K))$-term obtained by applying (13.1), so that

$$
\mathbf{S}(i /(K / s) t) p=\mathbf{S}(K / s) \mathbf{S}(i / t) p
$$

whenever $p$ is independent of $K$. Lemma (13.3) guarantees that the definition is unambiguous (i.e., that as long as $i$ satisfies the stated conditions, it does not matter how it is chosen). We proceed to derive some of the properties of the concept so defined. then

(13.5) Lemma. If $s$ is an $H$-term, $t$ is a $J$-term, and $K$ is a finite subset of $I$,

$$
(K / s) t=(J \cap K / s) t .
$$

Proof. Let $i$ be an element of $I-(H \cup J \cup K)$ and let $p$ be an element of $A$ independent of $K$. If $L$ is a finite support of $p$ such that $L \cap K=\varnothing$, then (by (7.5)) $J \cup L$ supports $\mathbf{S}(i / t) p$, and therefore

$$
\begin{aligned}
& \mathbf{S}(i /(K / s) t) p=\mathbf{S}(K / s) \mathbf{S}(i / t) p[\text { by (13.4)] } \\
& \quad=\mathbf{S}(K \cap(J \cup L) / s) \mathbf{S}(i / t) p[\text { by }(7.6)]=\mathbf{S}(J \cap K / s) \mathbf{S}(i / t) p[\text { since } L \cap K=\varnothing] \\
& \quad=\mathbf{S}(i /(J \cap K / s) t) p[\text { by (13.4)]; }
\end{aligned}
$$

the conclusion follows from (9.8).

Using (13.5), we can extend the definition of $(K / s) t$ to the case of a not necessarily finite $K$ simply by writing

$$
(K / s) t=(J \cap K / s) t .
$$


If $K$ happens to be finite, then the new definition of $(K / s) t$ is compatible with the old one. Observe that, since $J-K=J-(J \cap K)$, the term $(K / s) t$ is always an $(H \cup(J-K))$-term.

(13.6) Theorem. If $j$ is a variable, $t$ is a $J$-term, and $K$ is a subset of $I$, then

$$
\left(K / t_{j}\right) t=(K / j) t .
$$

Proof. By (13.5), $\left(K / t_{j}\right) t=\left(J \cap K / t_{j}\right) t$. Since the transformations $(K / j)$ and $(J \cap K / j)$ agree on $J$, it follows from (11.10) that $(K / j) t=(J \cap K / j) t$. These two comments together show that there is no loss of generality in assuming that the set $K$ is finite.

The transformation $(K / j)$ lives on $\{j\} \cup K$. If $i \epsilon I-(\{j\} \cup J \cup K)$ and if $p$ is independent of $\{j\} \cup K$, then

$$
\begin{aligned}
\mathbf{S}(i /(K / j) t) p & =\mathbf{S}(K / j) \mathbf{S}(i / t) p[\text { by (11.8)] } \\
& =\mathbf{S}\left(K / t_{j}\right) \mathbf{S}(i / t) p\left[\text { by (10.9)] }=\mathbf{S}\left(i /\left(K / t_{j}\right) t\right) p \quad[\text { by (13.4) }] .\right.
\end{aligned}
$$

The conclusion now follows from the uniqueness theorem (9.8).

(13.7) THEOREM. If $s$ is a term, if $\tau$ is a transformation, and if $k$ is an element of $I$ such that $\tau^{-1}\{k\}=\{k\}$, then

$$
(k / \tau s) \tau=\tau(k / s) .
$$

REMARK. The conclusion means that if $t$ is a term, then $(k / \tau s)(\tau t)$ $=\tau((k / s) t)$. Recall that if $j \in I$, and if $k$ and $\tau$ are as in the theorem, then $(k / \tau j) \tau=\tau(k / j)$.

Proof. Suppose that $s$ is an $H$-term, and let $t$ be a $J$-term. If $\tau_{0}$ is defined by writing $\tau_{0}=\tau$ on $H \cup J$ and $\tau_{0}=\delta$ outside $H \cup J$, then $\tau_{0}$ is a finite transformation, $\tau_{0}^{-1}\{k\}=\{k\}$, and, in view of (11.13), the desired conclusion $(k / \tau s) \tau t=\tau(k / s) t$ is equivalent to the corresponding equation for $\tau_{0}$. We may therefore assume that $\tau$ is finite in the first place.

Let $K$ be a finite set containing $k$ such that $\tau$ lives on $K$, and let $i$ be an element of $I-(H \cup J \cup \tau H \cup \tau J \cup K)$. If $p$ is independent of $K$, then

$$
\begin{aligned}
\mathbf{S}(i /(k / \tau s) \tau t) p & =\mathbf{S}(k / \tau s) \mathbf{S}(i / \tau t) p[\text { by }(13.4)] \\
= & \mathbf{S}(k / \tau s) \mathbf{S}(\tau) \mathbf{S}(i / t) p[\text { by }(11.8)]=\mathbf{S}(\tau) \mathbf{S}(k / s) \mathbf{S}(i / t) p[\text { by }(11.15)] \\
= & \mathbf{S}(\tau) \mathbf{S}(i /(k / s) t) p[\text { by }(13.4)]=\mathbf{S}(i / \tau(k / s) t) p[\text { by }(11.8)] .
\end{aligned}
$$

The conclusion follows from the uniqueness theorem (9.8).

(13.8) CoRollary. If $s$ is an H-term, if $\tau$ is a transformation such that $\tau=\delta$ on $H$, and if $k$ is an element of $I$ such that $\tau^{-1}\{k\}=\{k\}$, then

$$
(k / s) \tau=\tau(k / s) \text {. }
$$

REMARK. As in (13.7), the conclusion means that $(k / s)(\tau t)=\tau((k / s) t)$ for every term $t$. 
Proof. Apply (11.13) and (13.8).

(13.9) Theorem. If $s$ is an $H$-term, $t$ is a $J$-term, $K$ is a finite subset of $I$, and $\sigma$ is a transformation of type $(K, H \cup J)$, then

$$
(K / s) t=(\sigma K / s) \sigma t \text {. }
$$

Proof. Let $i$ be an element of $I-(H \cup J \cup K \cup \sigma J \cup \sigma K)$. If $p$ is an element of $\boldsymbol{A}$ such that $p$ is independent of $K \cup \sigma K$, then

$$
\begin{aligned}
\mathbf{S}(i /(\sigma K / s) \sigma t) p & =\mathbf{S}(\sigma K / s) \mathbf{S}(i / \sigma t) p[\text { by (13.4)] } \\
& =\mathbf{S}(\sigma K / s) \mathbf{S}(\sigma) \mathbf{S}(i / t) p[\text { by }(11.8)]=\mathbf{S}(K / s) \mathbf{S}(i / t) p[\text { by (4.1)] } \\
& =\mathbf{S}(i /(K / s) t) p[\text { by (13.4)]. }
\end{aligned}
$$

The conclusion follows from the uniqueness theorem (9.8).

(13.10) CoRollary. If $s$ is an H-term, $t$ is a J-term, $k$ is an element of $I$, and $i$ is an element of $I-(H \cup J)$, then

$$
(k / s) t=(i / s)(k / i) t .
$$

Proof. Apply (13.9) with $K=\{k\}$ and $\sigma=(k / i)$.

14. Terms in operations. Suppose that $T$ is an $n$-place operation and that $t_{1}, \cdots, t_{n}$ are terms; the purpose of this section is to show how it is possible to assign a sensible meaning to the expression $T\left(t_{1}, \cdots, t_{n}\right)$. The theory is similar to the corresponding part of the theory of terms in predicates.

(14.1) Lemma. Suppose that $J_{0}, J_{1}, \cdots, J_{n}$ are finite subsets of $I$, and that $t_{0}$ is a $J_{0}$-term, $t_{1}$ is a $J_{1}$-term, $\cdots, t_{n}$ is a $J_{n}$-term; write $J=J_{1} \cup \cdots \cup J_{n}$. If $i_{1}, \cdots, i_{n}, k_{1}, \cdots, k_{n}$ are $2 n$ distinct variables in $I-J$, and if $\left\{i_{1}, \cdots, i_{n}\right\}$ c $I-J_{0}$, then

$$
\left(i_{1} / t_{1}\right) \cdots\left(i_{n} / t_{n}\right)\left(k_{1} / i_{1}\right) \cdots\left(k_{n} / i_{n}\right) t_{0}=\left(k_{1} / t_{1}\right) \cdots\left(k_{n} / t_{n}\right) t_{0} .
$$

Proof. If $n \neq 1$, then (13.8) (with $s=t_{n}, \tau=\left(k_{1} / i_{1}\right)$, and $k=i_{n}$ ) implies that $\left(i_{n} / t_{n}\right)\left(k_{1} / i_{1}\right)=\left(k_{1} / i_{1}\right)\left(i_{n} / t_{n}\right)$. After a repeated application of this reasoning, the desideratum reduces to

$$
\left(i_{1} / t_{1}\right)\left(k_{1} / i_{1}\right) \cdots\left(i_{n} / t_{n}\right)\left(k_{n} / i_{n}\right) t_{0}=\left(k_{1} / t_{1}\right) \cdots\left(k_{n} / t_{n}\right) t_{0} .
$$

Corollary (13.10) implies that

$$
\left(i_{n} / t_{n}\right)\left(k_{n} / i_{n}\right) t_{0}=\left(k_{n} / t_{n}\right) t_{0} .
$$

The term $\left(k_{n} / t_{n}\right) t_{0}$ is a $\left(J_{n} \cup J_{0}\right)$-term, and $\left\{i_{1}, \cdots, i_{n}\right\} \subset I-\left(J_{n} \cup J_{0}\right)$. Since this comment prepares the ground for the induction step, (14.3) follows from an $n$-fold application of (13.10).

(14.4) Lemma. Suppose that $J_{1}, \cdots, J_{n}$ are finite subsets of $I$, that $t_{1}$ is a $J_{1}$-term, $\cdots, t_{n}$ is a $J_{n}$-term, and that $T$ is an $n$-place operation; write $J$ $=J_{1} \cup \cdots \cup J_{n}$. If $i_{1}, \cdots, i_{n}$ are distinct variables in $I-J$, and $j_{1}, \cdots, j_{n}$ 
are distinct variables in $I-J$, then

$$
\left(i_{1} / t_{1}\right) \cdots\left(i_{n} / t_{n}\right) T\left(i_{1}, \cdots, i_{n}\right)=\left(j_{1} / t_{1}\right) \cdots\left(j_{n} / t_{n}\right) T\left(j_{1}, \cdots, j_{n}\right) .
$$

Proof. Let $k_{1}, \cdots, k_{n}$ be distinct variables in $I-\left(J \cup\left\{i_{1}, \cdots, i_{n}\right\}\right.$ $\left.\cup\left\{j_{1}, \cdots, j_{n}\right\}\right)$ and write $T\left(k_{1}, \cdots, k_{n}\right)=t_{0}$. Since $\left(k_{1} / i_{1}\right) \cdots\left(k_{n} / i_{n}\right)$ $=\left(k_{1}, \cdots, k_{n} / i_{1}, \cdots, i_{n}\right)$, it follows from (12.3) that

$$
T\left(i_{1}, \cdots, i_{n}\right)=\left(k_{1} / i_{1}\right) \cdots\left(k_{n} / i_{n}\right) t_{0} .
$$

By (14.6) and (14.1), the left side of (14.5) is equal to $\left(k_{1} / t_{1}\right) \cdots\left(k_{n} / t_{n}\right) t_{0}$; the same argument with $j_{1}, \cdots, j_{n}$ in place of $i_{1}, \cdots, i_{n}$ shows that the right side of (14.5) is equal to the same thing.

If $T$ is an $n$-place operation, and if $t_{1}$ is a $J_{1}$-term, $\cdots, t_{n}$ is a $J_{n}$-term, we define $T\left(t_{1}, \cdots, t_{n}\right)$ by writing

$$
T\left(t_{1}, \cdots, t_{n}\right)=\left(i_{1} / t_{1}\right) \cdots\left(i_{n} / t_{n}\right) T\left(i_{1}, \cdots, i_{n}\right)
$$

whenever $i_{1}, \cdots, i_{n}$ are distinct variables in $I-\left(J_{1} \cup \cdots \cup J_{n}\right)$. Lemma (14.4) guarantees that this is an unambiguous definition.

(14.8) THEOREM. If $T$ is an $n$-place operation, if $t_{1}, \cdots, t_{n}$ are terms, and if $\tau$ is a transformation, then

$$
\tau T\left(t_{1}, \cdots, t_{n}\right)=T\left(\tau t_{1}, \cdots, \tau t_{n}\right) .
$$

Proof. Suppose that $t_{1}$ is a $J_{1}$-term, $\cdots, t_{n}$ is a $J_{n}$-term, and write $J=J_{1} \cup \cdots \cup J_{n}$. Let $\tau_{0}$ be the transformation that agrees with $\tau$ on $J$ and that is equal to $\delta$ outside $J$. Since the desired conclusion is the same for $\tau$ as for $\tau_{0}$, we may henceforth assume that the transformation $\tau$ is finite to begin with.

If $i_{1}, \cdots, i_{n}$ are distinct elements of $I-(J \cup \tau J)$, then $\tau^{-1}\left\{i_{1}\right\}=\left\{i_{1}\right\}$, $\cdots, \tau^{-1}\left\{i_{n}\right\}=\left\{i_{n}\right\}$, and therefore

$$
\begin{aligned}
\tau T\left(t_{1}, \cdots, t_{n}\right) & =\tau\left(i_{1} / t_{1}\right) \cdots\left(i_{n} / t_{n}\right) T\left(i_{1}, \cdots, i_{n}\right)[\text { by }(14.7)] \\
& =\left(i_{1} / \tau t_{1}\right) \cdots\left(i_{n} / \tau t_{n}\right) \tau T\left(i_{1}, \cdots, i_{n}\right)[\text { by }(13.7)] \\
& =\left(i_{1} / \tau t_{1}\right) \cdots\left(i_{n} / \tau t_{n}\right) T\left(i_{1}, \cdots, i_{n}\right)[\text { by }(12.3)] \\
& =T\left(\tau t_{1}, \cdots, \tau t_{n}\right) .
\end{aligned}
$$

We conclude this discussion of terms in operations by observing that there is a natural way of combining operations with other operations to form operations of higher degree. If $T$ is an operation of degree $n$, and if $T_{1}, \cdots, T_{n}$ are operations of degrees $m_{1}, \cdots, m_{n}$ respectively, we write $m=m_{1}+\cdots$ $+m_{n}$, and we define an operation $T\left(T_{1}, \cdots, T_{n}\right)$ of degree $m$ by substituting $T_{1}, \cdots, T_{n}$ into $T$. The precise details can be obtained by an obvious modification of the corresponding discussion in $\S 12$.

University of Chicago,

Chicago, Ill. 